\title{
Invariant see-saw models and sequential dominance
}

\author{
S. F. King ${ }^{1}$ \\ School of Physics and Astronomy, University of Southampton, \\ Southampton, SO17 1BJ, U.K.
}

\begin{abstract}
We propose an invariant see-saw (ISS) approach to model building, based on the observation that see-saw models of neutrino mass and mixing fall into basis invariant classes labelled by the Casas-Ibarra $R$-matrix, which we prove to be invariant not only under basis transformations but also non-unitary right-handed neutrino transformations $S$. According to the ISS approach, given any see-saw model in some particular basis one may determine the invariant $R$ matrix and hence the invariant class to which that model belongs. The formulation of see-saw models in terms of invariant classes puts them on a firmer theoretical footing, and allows different see-saw models in the same class to be related more easily, while their relation to the $R$-matrix makes them more easily identifiable in phenomenological studies. We also present an ISS mass formula which may be useful in model building. To illustrate the ISS approach we show that sequential dominance (SD) models form basis invariant classes in which the $R$-matrix is approximately related to a permutation of the unit matrix, and quite accurately so in the case of constrained sequential dominance (CSD) and tri-bimaximal mixing. Using the ISS approach we discuss examples of models in which the mixing naturally arises (at least in part) from the charged lepton or right-handed neutrino sectors and show that they are in the same invariant class as SD models. We also discuss the application of our results to flavour-dependent leptogenesis where we show that the case of a real $R$ matrix is approximately realized in SD, and accurately realized in CSD.
\end{abstract}

\footnotetext{
${ }^{1}$ E-mail: sfk@hep.phys.soton.ac.uk
} 


\section{Introduction}

The discovery and subsequent study of neutrino masses and mixing [1] remains the greatest advance in physics over the past decade. The latest experimental data [2] is consistent with (approximate) tri-bimaximal mixing [3] corresponding to $\sin \theta_{23} \approx 1 / \sqrt{2}$, $\sin \theta_{12} \approx 1 / \sqrt{3}, \sin \theta_{13} \approx 0[3$. How to incorporate small neutrino masses and large mixings into some new theory of flavour beyond the Standard Model has been the topic of intense theoretical activity [4] over the same period.

One particulary attractive mechanism is the see-saw mechanism [5], based on a simple extension of the (possibly Supersymmetric) Standard Model involving more than one right-handed neutrino $\nu_{R}$, coupling to left-handed lepton doublets $L$ with a matrix of "typical" Yukawa couplings $Y_{L R}^{\nu}$ (where "typical" means in the same ball park as the charged lepton Yukawa couplings $Y_{L R}^{E}$ of $L$ to right-handed charged leptons $E_{R}$ ) and having large (compared to the weak scale) Majorana masses $M_{R R}$. From these high energy inputs one may derive the low energy effective neutrino mass matrix from the see-saw formula $m_{L L}^{\nu}=v_{u}^{2} Y_{L R}^{\nu} M_{R R}^{-1} Y_{L R}^{\nu T}$ where $v_{u}$ is a Higgs vacuum expectation value $(\mathrm{VEV})$. From $m_{L L}^{\nu}$ and $Y_{L R}^{E}$ one may then obtain the low energy charged lepton masses $m_{e}, m_{\mu}, m_{\tau}$ and neutrino masses $m_{i}$ from the eigenvalues of the matrices, and $V_{M N S}^{\prime}=V_{E_{L}} V_{\nu_{L}}^{\dagger}$, where $V_{E_{L}}$ and $V_{\nu_{L}}$ diagonalize $Y_{L R}^{E}$ and $m_{L L}^{\nu}$ from the left. After non-physical phases are removed, the lepton mixing matrix $V_{M N S}$ can be compared to experiment.

There has been much theoretical effort devoted to understanding the origin and pattern of the high energy "see-saw" matrices $Y_{L R}^{\nu}, Y_{L R}^{E}$ and $M_{R R}$ which can lead to agreement with low energy data, via the see-saw mechanism [4. This problem is often considered together with the analogous one of the quark Yukawa matrices $Y_{L R}^{U}, Y_{L R}^{D}$, and is referred to as the "flavour problem". Although the flavour problem has been around for many years, the recent neutrino data provides additional challenges and constraints which have provided new insights into the problem, and a renewed impetus to attack it, resulting in an explosion of recent theoretical work in this direction. While it is impossible to review all the different models that have been proposed, the different approaches may be classified as either "kinematical" or "dynamical". In both the "kinematical" or "dynamical" approaches the goal is to "guess" or "derive" the input high energy input quark Yukawa $Y_{L R}^{U}, Y_{L R}^{D}$ and lepton see-saw matrices $Y_{L R}^{\nu}, Y_{L R}^{E}$ and $M_{R R}$. However, as has long been emphasized by Jarlskog [6] such matrices are not physical, since their appearance changes depending on the particular basis of underlying fields one chooses to work, and so working in a particular basis is meaningless.

This paper starts from the simple observation that not all choices of see-saw matrices $Y_{L R}^{\nu}, Y_{L R}^{E}$ and $M_{R R}$ which are consistent with a given set of low energy lepton parameters $m_{e}, m_{\mu}, m_{\tau}, m_{i}$ and $V_{M N S}$, are related to each other under a change of basis. This is in

contrast to the quark sector where all choices of Yukawa matrices $Y_{L R}^{U}, Y_{L R}^{D}$ consistent with a given set of low energy quark parameters $m_{u}, m_{c}, m_{t}, m_{d}, m_{s}, m_{b}$ and $V_{C K M}$, 
are related to each other under a change of basis. It is also in contrast to the effective lepton sector, where all choices of effective lepton matrices $m_{L L}^{\nu}$ and $Y_{L R}^{E}$ which are consistent with a given set of low energy lepton parameters $m_{e}, m_{\mu}, m_{\tau}, m_{i}$ and $V_{M N S}$, are related to each other under a change of basis. This observation implies that sets of see-saw matrices fall into invariant classes of models, $\left\{Y_{L R}^{\nu}, Y_{L R}^{E}, M_{R R}\right\} \in \mathcal{C}(R)$, where each different class $\mathcal{C}(R)$ is labelled by some continuous parameters $R$, where members of $\mathcal{C}(R)$ are consistent with the same low energy lepton observables $m_{e}, m_{\mu}, m_{\tau}, m_{i}$ and $V_{M N S}$, for all $R$. The set of all see-saw matrices within a particular invariant class $\mathcal{C}\left(R_{1}\right)$ are related to each other under a change of basis, but are not related to those in a different class $\mathcal{C}\left(R_{2}\right)$.

It is well known amongst the "phenomenological" community that the $R$ matrix of Casas and Ibarra [7] may be used to parameterize choices of see-saw matrices $Y_{L R}^{\nu}, Y_{L R}^{E}, M_{R R}$ consistent with a given set of low energy lepton parameters $m_{e}, m_{\mu}, m_{\tau}, m_{i}$ and $V_{M N S}$. Although it was appreciated by Casas and Ibarra that the $R$ matrix parameterization may be used in different lepton bases [7], this feature is rarely or never used in phenomenological analyses where people invariably work in the "flavour" basis where $Y_{L R}^{E^{\prime}}, M_{R R}^{\prime}$ are both diagonal. On the other hand, the $R$ matrix is largely ignored by the "theoretical" community who are concerned with guessing or deriving the see-saw matrices in a particular basis, which in general will not correspond to the "flavour" basis, so the $R$ matrix is not regarded as relevant.

In the present paper we show that the $R$ matrix is a basis invariant quantity, then propose using it in the context of model building to label the invariant class $\mathcal{C}(R)$ of see-saw models to which a particular model example belongs. Given a particular seesaw model there are several reasons why it is worth determining the invariant class $\mathcal{C}(R)$ to which it belongs, i.e. finding the invariant $R$ matrix associated with the particular see-saw model:

1. It puts the theory on a firmer theoretical foundation, since invariant quantities are always preferred to basis dependent one [6].

2. Given the $R$ matrix one may immediately generate an infinite set of equivalent seesaw models filling out the invariant class $\mathcal{C}(R)$ by applying lepton basis changes. This applies both to the "kinematical" and the "dynamical" approaches. So for any particular model (infinitely) many other models come for free.

3. It may turn out that a particular model under consideration corresponds to the same $R$ matrix as another model, i.e. the two models are in the same invariant class, in which case the two models should essentially must be regarded as the same model.

4. For given (class of) models, with $R$ specified one may immediately make contact with phenomenological analyses which have been performed in the literature which are relevant to testing the (class of) models. 
In this paper we shall illustrate the power of such an invariant see-saw (ISS) approach by discussing the case of sequential dominance (SD) [8]. SD is motivated by two considerations:

- To account for a neutrino mass hierarchy $m_{1} \ll m_{2} \ll m_{3}$ and large atmospheric and solar mixing angles in a natural way, without any tunings or cancellations. Although the $(2,3)$ mass hierarchy in the neutrino sector is not that strong, $m_{2} / m_{3} \approx 0.2$, we would still like to have a natural explanation for the smallness of this hierarchy, just as we would like to have an explanation for the smallness of the Cabibbo angle which has a similar value.

- To disentangle the question of the neutrino masses and the mixing angles, and so enable some explanation for tri-bimaximal neutrino mixing which involves elements in the MNS matrix having values equal to square roots of simple rational numbers such as $1 / 2$ or $1 / 3$. This would not be possible if the neutrino masses played a part in the calculation of the solar and atmospheric mixing angles.

In $\mathrm{SD}$, a natural neutrino mass hierarchy, $m_{2} / m_{3} \approx 0.2$, results from having one of the right-handed neutrinos give the dominant contribution to the see-saw mechanism, while a second right-handed neutrino gives the leading sub-dominant contribution, leading to a neutrino mass matrix with naturally small determinant [8]. ${ }^{1}$ In a basis where the righthanded neutrino mass matrix is diagonal, the atmospheric and solar neutrino mixing angles are determined in terms of ratios of Yukawa couplings involving the dominant and subdominant right-handed neutrinos, respectively. If these Yukawa couplings are related in a certain way, then it is possible for tri-bimaximal neutrino mixing, to emerge in a simple and natural way, independently of the neutrino mass eigenvalues. This is known as constrained sequential dominance (CSD) [10], and can readily arise from vacuum alignment in flavour models [10, 11, 12. In such unified flavour models there are corrections to tri-bimaximal mixing from charged lepton corrections, resulting in testable predictions and sum rules for lepton mixing angles [10, 13.

Although well motivated on physical grounds, SD appears to be restricted to a particular basis, namely that in which the right-handed neutrino and charged lepton mass matrices are both diagonal, although in particular model realizations there are typically small off-diagonal elements in both these mass matrices which must be taken into account. This might lead one to conclude that the notion of SD is quite limited, and furthermore that it is not physical since physical quantities should be basis independent. However, following the ISS approach advocated above, we will determine the invariant classes $\mathcal{C}(R)$ to which SD models belong, by finding the invariant $R$ matrix associated with each of the SD types, and hence show that SD can be formulated in a basis invariant way. In particular tri-bimaximal neutrino mixing from constrained SD is shown to have

\footnotetext{
${ }^{1}$ For alternative approaches involving a small determinant see 9 .
} 
an easily identifiable form in which the R-matrix is related to the unit matrix, where this form is preserved under charged lepton or right-handed neutrino basis changes, though the former gives observable corrections to the MNS parameters. Having done this we shall then reap the benefits mentioned above. Namely we shall show how certain models that have been proposed in the literature are equivalent to SD under a basis change, for example models where the mixing is completely or in part originating from the right-handed neutrino or charged lepton sectors [12, 14. We shall also discuss phenomenological analyses based on choices of $R$ matrix parameters that are seen to be relevant for SD.

In detail, the material discussed in this paper is structured as follows. In section 2 we discuss the ISS approach to model building that we advocate. We first review the well known result that all pairs of quark Yukawa matrices $Y_{L R}^{U}, Y_{L R}^{D}$ consistent with given physical parameters are related by basis transformations [6], and then show that a similar result holds for the effective lepton matrices $m_{L L}^{\nu}$ and $Y_{L R}^{E}$. We then show that a similar result does not apply to the see-saw mechanism, which leads to the notion of invariant classes of see-saw models, which may be parameterized by the $R$-matrix of Casas and Ibarra. We show how the $R$ matrix may be obtained and prove its invariance under basis transformations. We also propose a short-cut to obtaining the $R$ matrix, using a non-unitary $S$ matrix transformation of right-handed neutrinos, which is useful when right-handed neutrino mass eigenvalues are not required. In section 3 we discuss SD models as a prime example of the ISS approach. We first discuss this in a two family example, where a convenient vector notation for SD is introduced, and a relation between the $R$ matrix angle and the angle between these vectors is established. We then go on to the full three family case where we discuss the form of the $R$ matrix for all the types of $\mathrm{SD}$, and provide a systematic discussion of the $R$ matrix in the two righthanded neutrino limit in each case. Having established the relation between SD and the $R$ matrix, this then defines the invariant classes of see-saw models to which SD models belong, and hence allows the full set of models in these classes to be constructed by basis transformations. In section 4 we discuss the physical applications of these results to invariant classes of SD models. The particular forms of $R$ matrix associated with CSD and tri-bimaximal neutrino mixing are identified. SD is shown to be in the same invariant class as some models where the mixing completely or partly originates from the righthanded neutrino or charged lepton sectors [12, 14. We also discuss phenomenological analyses based on choices of $R$ matrix parameters that are seen to be relevant for SD. For example we discuss the application of our results to flavour-dependent leptogenesis [18, and show that the case of the real $R$ matrix may be (approximately) realized in SD. Section 5 concludes the paper.

Finally we would like to mention some earlier works where the relation between SD and the $R$ matrix has been mentioned before [15. We emphasize that the systematic discussion in this paper goes well beyond the nice discussions in [15], where the invariance of SD was not addressed. Moreover the ISS model building approach we advocate here, 
whereby any proposed see-saw model should be expressed in terms of the invariant $R$ matrix, represents a new strategy than can and should be applied to all see-saw models, not just the ones which satisfy SD as discussed here. The idea in this paper is to use the $R$ matrix more actively in model building (rather than in phenomenology, where it has been used extensively by many authors), with the hope that model builders will express their see-saw models in terms of the $R$ matrix (not normally done). The essential point of this paper is to emphasise that the $R$ matrix is invariant under basis transformations, since this feature, although clearly known by the inventors, is not so well used. It is precisely this invariance that means that the $R$ matrix can and should be more widely used as a model building tool, to classify and relate models.

\section{The ISS Model Building Approach}

\subsection{Quark sector}

In the quark sector the Dirac mass matrices of the up and down quarks are given by $m_{L R}^{U}=Y_{L R}^{U} v_{u}$, and $m_{L R}^{D}=Y_{L R}^{D} v_{d}$ where $v_{u}=\left\langle H_{u}^{0}\right\rangle$ and $v_{d}=\left\langle H_{d}^{0}\right\rangle$, and the Lagrangian is of the form $\mathcal{L}=-\bar{\psi}_{L} Y_{L R} H \psi_{R}+H$.c. The change from flavour basis to mass eigenstate basis can be performed with the unitary diagonalization matrices $V_{U_{L}}, V_{U_{R}}$ and $V_{D_{L}}, V_{D_{R}}$ by

$$
V_{U_{L}} m_{L R}^{U} V_{U_{R}}^{\dagger}=\operatorname{diag}\left(m_{u}, m_{c}, m_{t}\right), \quad V_{D_{L}} m_{L R}^{D} V_{D_{R}}^{\dagger}=\operatorname{diag}\left(m_{d}, m_{s}, m_{b}\right) .
$$

The CKM mixing matrix is then obtained from

$$
V_{C K M}^{\prime}=V_{U_{L}} V_{D_{L}}^{\dagger}
$$

where quark phase rotations which leave the quark masses real and positive may be used to remove five of the phases leaving one physical phase in the CKM matrix $V_{C K M}$. The Standard Model quark sector clearly respects the symmetry

$$
G_{\text {quark }}=U_{Q}(3) \times U_{U_{R}}(3) \times U_{D_{R}}(3)
$$

corresponding to quark doublet, right-handed up quark and right-handed down quark rotations, which change the quark basis and the form of the Yukawa matrices, but leave the physics (quark masses and mixings) unchanged. In the quark sector it is well known that the only physical quantities are basis independent invariants formed from the mass matrices, the so-called Jarlkog invariants [6], rather than the mass matrices themselves, since any pair of quark mass matrices which lead to the correct physics may be related to any other pair which lead to the same physics, by a change of basis, up to quark phases, using the symmetry $G_{\text {quark }}$.

This can be proved, for example, by showing that any two pairs of quark mass matrices can be related by a change of basis, using the symmetry $G_{\text {quark }}$, to a common 
basis in which the up quark mass matrix is diagonal, and the down quark mass matrix is equal, up to quark phases, to the CKM matrix multiplied by a diagonal matrix of down quark masses,

$$
m_{L R}^{U}{ }^{\prime}=\operatorname{diag}\left(m_{u}, m_{c}, m_{t}\right), \quad m_{L R}^{D}{ }^{\prime}=V_{C K M}^{\prime} \operatorname{diag}\left(m_{d}, m_{s}, m_{b}\right) .
$$

Since any two pairs of mass matrices $\left(m_{L R}^{U}\right)_{1},\left(m_{L R}^{D}\right)_{1}$ and $\left(m_{L R}^{U}\right)_{2},\left(m_{L R}^{D}\right)_{2}$ may be related to $m_{L R}^{U}{ }^{\prime}, m_{L R}^{D}{ }^{\prime}$ in Eq4 by a change of basis, it follows that all choices of quark mass matrices which lead to the same physics can be related to each other, up to quark phases, using the symmetry $G_{\text {quark }}$. This implies that the quark mass matrices $m_{L R}^{U}$, $m_{L R}^{D}$ are not physical quantities since they are basis dependent, i.e. not invariant under the symmetry $G_{\text {quark }}$. It is possible to define $G_{\text {quark }}$ invariant combinations consisting of determinants and traces of products of the combinations $S_{L L}^{U}=m_{L R}^{U}\left(m_{L R}^{U}\right)^{\dagger}$ and $S_{L L}^{D}=m_{L R}^{D}\left(m_{L R}^{D}\right)^{\dagger}$, for example the determinant of the commutator $\operatorname{det}\left[S_{L L}^{U}, S_{L L}^{D}\right]$ is an invariant [6].

\subsection{Effective lepton sector}

From the point of view of low energy neutrino experiments, Majorana neutrino masses arise from the effective operator: $\mathcal{L}^{e f f}=-\frac{1}{2} H_{u} L^{T} \kappa H_{u} L+H . c$. where $L$ are the lepton doublets, $H_{u}$ are Higgs doublets, and $\kappa$ is a matrix of effective (dimensional) couplings. In our convention the effective Majorana masses are given by the Lagrangian $\mathcal{L}=$ $-\bar{\nu}_{L} m_{L L}^{\nu} \nu^{c}+H . c$. where $m_{L L}^{\nu}=\kappa^{*} v_{u}^{2}$. The rotation to the mass eigenstate basis can be performed with the unitary diagonalization matrices $V_{E_{L}}, V_{E_{R}}$ and $V_{\nu_{L}}$ by

$$
V_{E_{L}} m_{L R}^{E} V_{E_{R}}^{\dagger}=\operatorname{diag}\left(m_{e}, m_{\mu}, m_{\tau}\right), \quad V_{\nu_{L}} m_{L L}^{\nu} V_{\nu_{L}}^{T}=\operatorname{diag}\left(m_{1}, m_{2}, m_{3}\right) .
$$

The lepton mixing matrix is then obtained from

$$
V_{M N S}^{\prime}=V_{E_{L}} V_{\nu_{L}}^{\dagger}
$$

where charged lepton phases rotations which leave the charged lepton masses real and positive may be used to remove three of the phases leaving three physical phases in the MNS matrix $V_{M N S}$.

The effective lepton sector clearly respects the symmetry

$$
G_{\text {lepton }}^{\text {eff }}=U_{L}(3) \times U_{E_{R}}(3)
$$

corresponding to lepton doublet and right-handed charged lepton rotations, which change the lepton basis and the form of the effective lepton matrices, but leave the physics (lepton masses and mixings) unchanged. The physically measurable low energy lepton parameters are the three charged lepton masses $m_{e}, m_{\mu}, m_{\tau}$, the three neutrino masses $m_{1,2,3}>0$ and the lepton mixing parameters contained in $V_{M N S}$. 
As in the quark sector, any pair of effective lepton matrices $m_{L R}^{E}, m_{L L}^{\nu}$ which lead to a given low energy physics may be related to any other pair which lead to the same physics, by a change of basis, using the symmetry $G_{\text {lepton }}^{\text {eff }}$. This is easily proved (analagous to the quark sector) by tranforming to a common basis in which the charged lepton mass matrix is diagonal, and the effective Majorana neutrino mass matrix is specified in terms of the lepton mixing matrix $V_{M N S}^{\prime}=V_{E_{L}} V_{\nu_{L}}^{\dagger}$ and the physical neutrino masses $m_{i}$,

$$
m_{L R}^{E}{ }^{\prime}=\operatorname{diag}\left(m_{e}, m_{\mu}, m_{\tau}\right), \quad m_{L L}^{\nu}{ }^{\prime}=V_{M N S}^{\prime} \operatorname{diag}\left(m_{1}, m_{2}, m_{3}\right) V_{M N S}^{T}
$$

where Eq 8, often called the "flavour basis", is analagous to Eq4. Then, as in the quark case, we can argue that since any two pairs of matrices $\left(m_{L R}^{E}\right)_{1},\left(m_{L L}^{\nu}\right)_{1}$ and $\left(m_{L R}^{E}\right)_{2}$, $\left(m_{L L}^{\nu}\right)_{2}$ can be rotated to the flavour basis then they can therefore be rotated into each other, using the symmetry $G_{\text {lepton }}^{\text {eff }}$, analagous to the quark sector result. $m_{L R}^{E}, m_{L L}^{\nu}$ are clearly basis dependent, but invariants under $G_{\text {lepton }}^{\text {eff }}$ can be constructed using $S_{L L}^{E}=$ $m_{L R}^{E}\left(m_{L R}^{E}\right)^{\dagger}$ and $S_{L L}^{\nu}=m_{L L}^{\nu}\left(m_{L L}^{\nu}\right)^{\dagger}$, for example the determinant of the commutator $\operatorname{det}\left[S_{L L}^{E}, S_{L L}^{\nu}\right]$ is invariant.

\subsection{See-saw sector}

The starting point of the see-saw mechanism is the Lagrangian,

$$
\mathcal{L}_{\text {seesaw }}=-Y_{L R}^{E} H_{d} \bar{L} E_{R}-Y_{L R}^{\nu} H_{u} \bar{L} \nu_{R}+\frac{1}{2} \nu_{R}^{T} M_{R R} \nu_{R}+H . c .
$$

where all indices have been suppressed, and we have introduced two Higgs doublets $H_{u}, H_{d}$ as in the Supersymmetric Standard Model. ${ }^{2}$ It is common to call Eq9 the seesaw Lagrangian. After integrating out the right-handed neutrinos it leads to an effective low energy leptonic Lagrangian of the type discussed in the previous subsection where the effective Majorana mass matrix given by the (type I) see-saw formula:

$$
m_{L L}^{\nu}=v_{u}^{2} Y_{L R}^{\nu} M_{R R}^{-1} Y_{L R}^{\nu T}
$$

The effective low energy matrices are diagonalised by unitary transformations $V_{E_{L}}, V_{E_{R}}$ and $V_{\nu_{L}}$ as in Eq 5 , and the lepton mixing matrix is as in Eq, 6 .

The lepton symmetry of the see-saw Lagrangian in Eq.9 is:

$$
G_{\text {lepton }}=U_{L}(3) \times U_{E_{R}}(3) \times U_{\nu_{R}}(3)
$$

corresponding to lepton doublet, right-handed charged lepton and right-handed neutrino rotations, which change the lepton basis and the form of the see-saw matrices, but leave the physics (lepton masses and mixings) unchanged. Using these symmetries we can

\footnotetext{
${ }^{2}$ In the case of the Standard Model one of the two Higgs doublets is equal to the charge conjugate of the other, $H_{d} \equiv H_{u}^{c}$.
} 
ask the question whether all sets of see-saw matrices $Y_{L R}^{E}, Y_{L R}^{\nu}$ and $M_{R R}$ which lead to a given set of low energy physical lepton parameters are equivalent to each other by a change of basis. Analagous to the quark sector, we may attempt to relate all sets of see-saw matrices to a common set of see-saw matrices in which the charged lepton mass matrix is diagonal, and the right-handed neutrino Majorana mass matrix is also diagonal,

$$
v_{d} Y_{L R}^{E^{\prime}}=\operatorname{diag}\left(m_{e}, m_{\mu}, m_{\tau}\right), \quad M_{R R}^{\prime}=\operatorname{diag}\left(M_{1}, M_{2}, M_{3}\right), \quad Y_{L R}^{\nu{ }^{\prime}}=V_{E_{L}} Y_{L R}^{\nu} V_{\nu_{R}}^{\dagger}
$$

where unitary $V_{\nu_{R}}$ is defined by $V_{\nu_{R}} M_{R R} V_{\nu_{R}}^{T}=M_{R R}^{\prime}$ and $M_{i}>0$.

We refer to the basis of Eq,12 as the "see-saw flavour basis" in analogy to Eq[8. The difference between Eqs 48 and Eq12 is that here $Y_{L R}^{\nu}{ }^{\prime}$ is not uniquely specified since it is diagonalized by left-handed rotations which are not simply related to the lepton mixing matrix, and in addition its eigenvalues are not simply related to physical neutrino masses. Therefore, unlike the quark sector, or the effective lepton case, there is not a unique common basis. Therefore, we conclude that any two sets of see-saw matrices $\left(Y_{L R}^{E}\right)_{1},\left(Y_{L R}^{\nu}\right)_{1},\left(M_{R R}\right)_{1}$ and $\left(Y_{L R}^{E}\right)_{2},\left(Y_{L R}^{\nu}\right)_{2},\left(M_{R R}\right)_{2}$ which give the same physical right-handed neutrino masses, light effective neutrino masses, charged lepton masses and lepton mixings, cannot be transformed into each other under the lepton see-saw symmetry $G_{\text {lepton }}$ corresponding to basis changes.

We note parenthetically that although the see saw formula is not a basis invariant, by taking its determinant one can obtain the invariant mass formula:

$$
m_{1} m_{2} m_{3}=\frac{m_{D_{1}}^{2} m_{D_{2}}^{2} m_{D_{3}}^{2}}{M_{1} M_{2} M_{3}}
$$

where $m_{i}$ are the physical light left-handed neutrino masses, $M_{i}$ are the heavy righthanded neutrino masses, and $m_{D_{i}}$ are the eigenvalues of the Dirac neutrino mass matrix $m_{L R}^{\nu}=v_{u} Y_{L R}^{\nu}$. Eq13 is, apparently, a new result which we could not find quoted in the literature. We shall refer to it as the ISS mass formula. The product of diagonal squared Dirac mass eigenvalues, is clearly an invariant since it is given by $\operatorname{det}\left(m_{L R}^{\nu} m_{L R}^{\nu}{ }^{\dagger}\right.$. Although Eq 13 should have useful see-saw model building applications with respect to neutrino masses, it clearly does not shed any light on the question of neutrino mixing.

\subsection{Invariant classes of see-saw models and the $R$-matrix}

We have seen that, in contrast to the case of the effective lepton or quark sector, not all choices of see-saw matrices $Y_{L R}^{\nu}, Y_{L R}^{E}$ and $M_{R R}$ which are consistent with a given set of low energy lepton parameters $m_{e}, m_{\mu}, m_{\tau}, m_{i}$ and $V_{M N S}$, are related to each other under a change of basis. This implies that sets of see-saw matrices fall into invariant classes of models, $\left\{Y_{L R}^{\nu}, Y_{L R}^{E}, M_{R R}\right\} \in \mathcal{C}(R)$, where each different class $\mathcal{C}(R)$ is labelled by some continuous parameters $R$, where members of $\mathcal{C}(R)$ are consistent with the 
same low energy lepton observables $m_{e}, m_{\mu}, m_{\tau}, m_{i}$ and $V_{M N S}$, for all $R$. The set of all see-saw matrices within a particular invariant class $\mathcal{C}\left(R_{1}\right)$ are related to each other under a change of basis, but are not related to those in a different class $\mathcal{C}\left(R_{2}\right)$. In this subsection we show that the $R$ matrix of Casas and Ibarra [7], which is well known in phenomenological applications, is a basis invariant quantity. We then propose using it in the context of model building to label the invariant class $\mathcal{C}(R)$ of see-saw models to which a particular model example belongs.

Following [7], we first derive the $R$ matrix in the see-saw flavour basis in Eq [12, by constraining $Y_{L R}^{\nu}{ }^{\prime}$ to give $m_{L L}^{\nu}{ }^{\prime}$ in the basis in Eq[8] using the see-saw mechanism in Eq[10.

$$
v_{u}^{2} Y_{L R}^{\nu}{ }^{\prime} \operatorname{diag}\left(M_{1}, M_{2}, M_{3}\right)^{-1} Y_{L R}^{\nu{ }^{\prime} T}=V_{M N S}^{\prime} \operatorname{diag}\left(m_{1}, m_{2}, m_{3}\right) V_{M N S}^{\prime} \cdot
$$

In order to solve Eq14 for the neutrino Yukawa matrix $Y_{L R}^{\nu}{ }^{\prime}$ we can try to write both sides of the equation in the form $A A^{T}=B B^{T}$ then take the positive square root of the equation to give,

$$
v_{u} Y_{L R}^{\nu}{ }^{\prime} \operatorname{diag}\left(M_{1}, M_{2}, M_{3}\right)^{-1 / 2}=V_{M N S}^{\prime} \operatorname{diag}\left(m_{1}, m_{2}, m_{3}\right)^{1 / 2} R^{T}
$$

where $R$ is the Casas-Ibarra complex orthogonal matrix, $R^{T} R=I$ where $I$ is the unit matrix. It is often used in phenomenological analyses to parameterize $Y_{L R}^{\nu}{ }^{\prime}$ in the see-saw flavour basis, since $R$ determines $Y_{L R}^{\nu}{ }^{\prime}$ in terms of physical parameters from Eq 15

In the above discussion the $R$ matrix was derived in the see-saw flavour basis. However one can repeat the above derivation starting from a general charged lepton basis in which neither $Y_{L R}^{E}$ nor $Y_{L R}^{\nu}$ (unprimed matrices) are in general not diagonal (but retaining for the moment a diagonal right-handed neutrino mass matrix) leading to:

$$
v_{u} Y_{L R}^{\nu} \operatorname{diag}\left(M_{1}, M_{2}, M_{3}\right)^{-1 / 2}=V_{\nu_{L}}^{\dagger} \operatorname{diag}\left(m_{1}, m_{2}, m_{3}\right)^{1 / 2} R^{T}
$$

where $V_{\nu_{L}}$ is the matrix that diagonalizes $m_{L L}^{\nu}$ in this basis, as in Eq 5 . Comparing Eq16 to Eq15 the only change is to left-hand sides of the equations, where in the seesaw flavour basis it happens that $V_{\nu_{L}}^{\dagger}=V_{M N S}^{\prime}$. The fact that the same $R$ matrix appears in Eq16] as Eq15 follows from the fact that $Y_{L R}^{\nu^{\prime}}=V_{E_{L}} Y_{L R}^{\nu}$, where $V_{E_{L}}$ diagonalizes the charged lepton mass matrix as in Eq 5. Therefore by multiplying on the left-hand sides of Eq.16 by $V_{E_{L}}$, and comparing the resulting equation to Eq15, where the MNS matrix is given by Eq, we find the non-trivial result that the same $R$ matrix must appear in both Eq 15 and Eq[16. We conclude that the $R$ matrix is invariant under a change of charged lepton basis.

We now prove that the $R$ matrix is invariant under a change of right-handed neutrino basis, so that the right-handed neutrinos are no longer diagonal. The main observation is that according to the $R$ matrix parameterizes only the combination on the left-hand side of Eq16, and this combination is clearly invariant under $U_{\nu_{R}}(3)$, which also preserves the right-handed neutrino masses. Under $\nu_{R} \rightarrow V_{\nu_{R}} \nu_{R}$, Eq16 thus becomes,

$$
v_{u} Y_{L R}^{\nu} V_{\nu_{R}}^{\dagger} \operatorname{diag}\left(M_{1}, M_{2}, M_{3}\right)^{-1 / 2}=V_{\nu_{L}}^{\dagger} \operatorname{diag}\left(m_{1}, m_{2}, m_{3}\right)^{1 / 2} R^{T}
$$


with $R$ again invariant. The invariance of the $R$ matrix, together with Eq[17 suggests the following ISS model building strategy. In some particular given basis where the see-saw matrices $Y_{L R}^{\nu}, Y_{L R}^{E}$ and $M_{R R}$ are not diagonal, Eq[17 may be used to determine the $R$ matrix in terms of the masses $m_{i}, M_{i}$, the matrix $V_{\nu_{L}}$ which diagonalises $m_{L L}^{\nu}$ in this basis, as in Eq [5, and $V_{\nu_{R}}$ as defined below Eq[12. Since the $R$ matrix is invariant under a change of basis, as we have shown, it may then be used to label invariant class of models to which the particular see-saw matrices belong, $\left\{Y_{L R}^{\nu}, Y_{L R}^{E}, M_{R R}\right\} \in \mathcal{C}(R)$.

Finally we show that the $R$ matrix is also invariant under non-unitary right-handed neutrino transformations, namely $\nu_{R} \rightarrow S \nu_{R}$, where $S$ is non-singular, which results in:

$$
Y_{L R}^{\nu} \rightarrow Y_{L R}^{\nu} S^{-1}, \quad M_{R R} \rightarrow S^{T^{-1}} M_{R R} S^{-1}, \quad M_{R R}^{-1} \rightarrow S M_{R R}^{-1} S^{T} .
$$

The transformations in Eq18 leave the effective low energy neutrino mass matrix $m_{L L}^{\nu}$ invariant, which follows from the see-saw mechanism in Eq10. However the right-handed neutrino masses will change, since $S$ is non-unitary. By a suitable choice of $S, M_{R R}$ can be transformed into a diagonal form,

$$
S^{T^{-1}} M_{R R} S^{-1}=\operatorname{diag}\left(\tilde{M}_{1}, \tilde{M}_{2}, \tilde{M}_{3}\right)
$$

where we emphasize that the choice of $S$ is not unique, and $\tilde{M}_{i}$ are not the eigenvalues of $M_{R R}$. For example, without loss of generality, $S$ can always be chosen so that $\tilde{M}_{i}$ are all equal to unity in some units. Allowing non-unitary $S$ matrix transformations, one can derive a similar result to Eq17.

$$
v_{u} Y_{L R}^{\nu} S^{-1} \operatorname{diag}\left(\tilde{M}_{1}, \tilde{M}_{2}, \tilde{M}_{3}\right)^{-1 / 2}=V_{\nu_{L}}^{\dagger} \operatorname{diag}\left(m_{1}, m_{2}, m_{3}\right)^{1 / 2} R^{T}
$$

where $S$ and $\tilde{M}_{i}$ are defined in Eq,19, and $V_{\nu_{L}}$ is as before since $m_{L L}^{\nu}$ is invariant under $S$ matrix transformations. $R$ is once again invariant, which essentially follows from the invariance of the combination on the left-hand side of Eq20 under $S$ matrix transformations. For a given non-diagonal set of see-saw matrices $Y_{L R}^{\nu}, Y_{L R}^{E}$ and $M_{R R}$, Eq20 can sometimes be used as a short-cut to determining the invariant $R$ matrix, instead of Eq[17. Since the $R$ matrix is invariant under the $S$ matrix, as we have shown, it may then be used to label invariant class of models to which the particular see-saw matrices belong, $\left\{Y_{L R}^{\nu}, Y_{L R}^{E}, M_{R R}\right\} \in \mathcal{C}(R)$, as before. The $S$ matrix approach may be especially useful in low energy applications where the right-handed neutrino masses are not required.

\section{ISS approach to SD}

\subsection{Two family SD in the see-saw flavour basis}

In this section we shall show that sequential dominance (SD) models [8] correspond to particular invariant classes of see-saw models characterized by particular forms of the 
R-matrix. SD provides a good example of the invariant see-saw (ISS) approach, since SD is sometimes criticized as being only valid in a special basis, namely the see-saw flavour basis. Defining SD in terms of the $R$ matrix renders the SD approach basis independent which overcomes this criticism, and brings with it all the benefits already mentioned previously, some of which will be explored further in the next section on Applications. We shall begin by discussing the dominance mechanism in a simple two family example, first in the see-saw flavour basis, then in terms of the $R$ matrix which defines a basis independent formulation of SD. We then extend this discussion to include three families, then take the two right-handed neutrino limit of these models.

To review the basic idea of SD, then, it is instructive to begin by discussing a simple $2 \times 2$ example applicable to the atmospheric mixing in the $(2,3)$ sector, in the see-saw flavour basis, i.e. the diagonal charged lepton and right-handed neutrino Majorana mass basis, where we can write,

$$
M_{R R}=\left(\begin{array}{cc}
M_{A} & 0 \\
0 & M_{B}
\end{array}\right), \quad m_{L R}^{\nu}=\left(\begin{array}{cc}
A_{2} & B_{2} \\
A_{3} & B_{3}
\end{array}\right)
$$

where $m_{L R}^{\nu}=Y_{L R}^{\nu} v_{u}$. It is sufficient for the toy model to ignore phases, and suppose that $A_{i}, B_{i}$ are real. The see-saw formula in Eq10 $m_{L L}^{\nu}=m_{L R}^{\nu} M_{R R}^{-1} m_{L R}^{\nu}$ gives:

$$
m_{L L}^{\nu}=\left(\begin{array}{cc}
\frac{A_{2}^{2}}{M_{A}}+\frac{B_{2}^{2}}{M_{B}} & \frac{A_{2} A_{3}}{M_{A}}+\frac{B_{2} B_{3}}{M_{B}} \\
\frac{A_{2} A_{3}}{M_{A}}+\frac{B_{2} B_{3}}{M_{B}} & \frac{A_{3}^{2}}{M_{A}}+\frac{B_{3}^{2}}{M_{B}}
\end{array}\right)
$$

The mass matrix in Eq22 is diagonalized to give two neutrino mass eigenvalues $m_{2}, m_{3}$ by rotating through an angle $\theta_{23}$ given by,

$$
\tan 2 \theta_{23}=\frac{2\left(\frac{A_{2} A_{3}}{M_{A}}+\frac{B_{2} B_{3}}{M_{B}}\right)}{\left(\frac{A_{2}^{2}}{M_{A}}+\frac{B_{2}^{2}}{M_{B}}\right)-\left(\frac{A_{2}^{2}}{M_{A}}+\frac{B_{2}^{2}}{M_{B}}\right)} .
$$

The determinant of the neutrino mass matrix $m_{L L}^{\nu}$ in $\mathrm{Eq} 22$ is

$$
\operatorname{det}_{L L}^{\nu}=\frac{1}{M_{A} M_{B}}\left(A_{2} B_{3}-A_{3} B_{2}\right)^{2}=m_{2} m_{3}
$$

and the trace of the neutrino mass matrix $m_{L L}^{\nu}$ in Eq,22 is

$$
\operatorname{Trm}_{L L}^{\nu}=\frac{A_{2}^{2}}{M_{A}}+\frac{A_{3}^{2}}{M_{A}}+\frac{B_{2}^{2}}{M_{B}}+\frac{B_{3}^{2}}{M_{B}}=m_{2}+m_{3} \approx m_{3}
$$

where the last approximation assumes a neutrino mass hierarchy $m_{3} \gg m_{2} \cdot m_{2}$ is then approximately determined from the trace and determinant of the mass matrix as,

$$
m_{2}=\frac{\operatorname{det}_{L L}^{\nu}}{m_{3}} \approx \frac{\operatorname{detm}_{L L}^{\nu}}{\operatorname{Trm}_{L L}^{\nu}} \approx \frac{\frac{\left(A_{2} B_{3}-A_{3} B_{2}\right)^{2}}{M_{A} M_{B}}}{\frac{\left(A_{2}^{2}+A_{3}^{2}\right)}{M_{A}}+\frac{\left(B_{2}^{2}+B_{3}^{2}\right)}{M_{B}}} .
$$


The basic assumption of SD is that one of the right-handed neutrinos plays the dominant role in the see-saw mechanism. Without loss of generality we shall assume that the right-handed neutrino of mass $M_{A}$ dominates the see-saw mechanism:

$$
\frac{\left|A_{i} A_{j}\right|}{M_{A}} \gg \frac{\left|B_{i} B_{j}\right|}{M_{B}}
$$

Assuming the dominance approximation in Eq 27, the determinant and trace of the mass matrix in Eq22 imply that the neutrino mass spectrum then consists of one neutrino with mass $m_{3} \approx\left(A_{2}^{2}+A_{3}^{2}\right) / M_{3}$ and one naturally light neutrino $m_{2} \ll m_{3}$ determined from $\mathrm{Eq} 26$, since the determinant of $\mathrm{Eq} 22$ is naturally small, and vanishes in the extreme limit of the dominance approximation when only one right-handed neutrino contributes [8]. Under the dominance approximation in Eq 27, the atmospheric angle from Eq 23 is $\tan \theta_{23} \approx A_{2} / A_{3}\left[8\right.$, which can be large or maximal providing $A_{2} \approx A_{3}$. Collecting together these results, the dominance approximation in Eq[27]leads to,

$$
m_{3} \approx \frac{\left(A_{2}^{2}+A_{3}^{2}\right)}{M_{A}}, \quad m_{2} \approx \frac{\left(A_{2} B_{3}-A_{3} B_{2}\right)^{2}}{\left(A_{2}^{2}+A_{3}^{2}\right) M_{B}}, \quad \tan \theta_{23} \approx \frac{A_{2}}{A_{3}} .
$$

Therefore, assuming the dominance of a single right-handed neutrino, Eq.28 shows that $m_{3}$ is determined approximately by the right-handed neutrino with mass $M_{A}, m_{2}$ is determined approximately by the right-handed neutrino with mass $M_{B}$, and $\tan \theta_{23}$ is determined approximately by a simple ratio of Yukawa couplings, independently of the neutrino mass hierarchy. Note that right-handed neutrino dominance allows the origin of the large mixing angle to be decoupled from the neutrino mass hierarchy, allowing both features to co-emerge in a very natural way.

The above results can be expressed more compactly by introducing the column vector notation,

$$
\underline{v_{A}}=\left(\begin{array}{c}
A_{2} \\
A_{3}
\end{array}\right) M_{A}^{-1 / 2}, \quad \underline{v_{B}}=\left(\begin{array}{c}
B_{2} \\
B_{3}
\end{array}\right) M_{B}^{-1 / 2}
$$

Then the see-saw formula in Eq[10 $m_{L L}^{\nu}=m_{L R}^{\nu} M_{R R}^{-1} m_{L R}^{\nu}$ gives:

$$
m_{L L}^{\nu}=\underline{v_{A}}{\underline{v_{A}}}^{T}+\underline{v_{B}}{\underline{v_{B}}}^{T}
$$

The determinant of the neutrino mass matrix $m_{L L}^{\nu}$ is

$$
\operatorname{det}_{L L}^{\nu}=\left|\underline{v_{A}} \times \underline{v_{B}}\right|^{2}=m_{2} m_{3}
$$

and the trace of the neutrino mass matrix $m_{L L}^{\nu}$ is

$$
\operatorname{Tr}_{L L}^{\nu}=\left|\underline{v_{A}}\right|^{2}+\left|\underline{v_{B}}\right|^{2}=m_{2}+m_{3} \approx m_{3}
$$


$m_{2}$ is then approximately determined from the trace and determinant of the mass matrix as,

$$
m_{2}=\frac{\operatorname{det}_{L L}^{\nu}}{m_{3}} \approx \frac{\operatorname{detm}_{L L}^{\nu}}{\operatorname{Trm}_{L L}^{\nu}} \approx \frac{\left|\underline{v_{A}} \times \underline{v_{B}}\right|^{2}}{\left|\underline{v_{A}}\right|^{2}+\left|\underline{v_{B}}\right|^{2}} .
$$

To arrange for a hierarchy $m_{2} / m_{3} \approx 1 / 5$, we require the determinant to be small compared to the square of the trace. This may be achieved using the dominance condition in Eq27 that the right-handed neutrino of mass $M_{A}$ gives the dominant contribution to the see-saw mechanism, which in vector notation implies:

$$
\left|\underline{v_{A}}\right|^{2} \gg\left|\underline{v_{B}}\right|^{2} \text {. }
$$

We shall see in the next section that the dominance approximation leads to the vectors $v_{A}$ and $v_{B}$ being approximately orthogonal and that there is a precise correlation between the degree of orthogonality of these two vectors and the degree of dominance. Here we give two examples which illustrate that the dominance condition only applies when the two vectors $\underline{v_{A}}$ and $\underline{v_{B}}$ are sufficiently orthogonal:

- If $A_{2}=A_{3}$ and $B_{2}=-B_{3}$, corresponding to the two vectors $\underline{v_{A}}$ and $\underline{v_{B}}$ being exactly orthogonal, then Eq28 gives,

$$
m_{3} \approx \frac{2 A_{2}^{2}}{M_{A}}, \quad m_{2} \approx \frac{2 B_{2}^{2}}{M_{B}}, \quad \tan \theta_{23} \approx 1 .
$$

and the required hierarchy $m_{2} / m_{3} \approx 1 / 5$ then implies that,

$$
\frac{A_{2}^{2}}{M_{A}} \approx 5 \frac{B_{2}^{2}}{M_{B}}
$$

which satisfies the dominance condition in Eq.27.

- Now suppose that the two vectors are at $45^{\circ}$ to each other, such as given by $A_{2}=A_{3}$, and $B_{3}=0$, then Eq 28 becomes,

$$
m_{3} \approx \frac{2 A_{2}^{2}}{M_{A}}, \quad m_{2} \approx \frac{B_{2}^{2}}{2 M_{B}}, \quad \tan \theta_{23} \approx 1
$$

and the required hierarchy $m_{2} / m_{3} \approx 1 / 5$ then implies that,

$$
\frac{A_{2}^{2}}{M_{A}} \approx \frac{5}{4} \frac{B_{2}^{2}}{M_{B}}
$$

which only marginally satisfies the dominance condition in Eq.27. If the vectors are more closely aligned than about $45^{\circ}$ then the dominance condition will not be satisfied. 


\subsection{Two family SD and the $R$ matrix}

According to the ISS approach, we should formulate SD in terms of the invariant Rmatrix. From Eq[15, we have for the two family toy model in Eq.21, dropping primes, and assuming $M_{A}<M_{B}$ :

$$
m_{L R}^{\nu}\left(\begin{array}{cc}
M_{A} & 0 \\
0 & M_{B}
\end{array}\right)^{-1 / 2}=V_{M N S}^{2 \times 2}\left(\begin{array}{cc}
m_{2} & 0 \\
0 & m_{3}
\end{array}\right)^{1 / 2} R_{2 \times 2}^{T} .
$$

The MNS matrix is parameterized by the atmospheric angle $\theta_{23}$, and the R-matrix may be parameterized here by an angle $\theta$, ignoring phases,

$$
V_{M N S}^{2 \times 2}=\left(\begin{array}{cc}
c_{23} & s_{23} \\
-s_{23} & c_{23}
\end{array}\right), \quad R_{2 \times 2}^{T}=\left(\begin{array}{cc}
c & -s \\
s & c
\end{array}\right) .
$$

where $c=\cos \theta, s=\sin \theta$. Each choice of $\theta$ specifies a particular solution to the see-saw formula for the combination $m_{L R}^{\nu} M_{R R}^{-1 / 2}$ on the left-hand side of Eq39.

Using Eqs 3940, we find the following expressions for the vectors introduced in Eqs 29.

$$
\begin{aligned}
& \underline{v_{A}}=c m_{2}^{1 / 2}\left(\begin{array}{c}
c_{23} \\
-s_{23}
\end{array}\right)+s m_{3}^{1 / 2}\left(\begin{array}{l}
s_{23} \\
c_{23}
\end{array}\right) \\
& \underline{v_{B}}=-s m_{2}^{1 / 2}\left(\begin{array}{c}
c_{23} \\
-s_{23}
\end{array}\right)+c m_{3}^{1 / 2}\left(\begin{array}{l}
s_{23} \\
c_{23}
\end{array}\right) .
\end{aligned}
$$

The single right-handed neutrino dominance approximation in Eq 34 is then seen from Eq41 to correspond to values of $\theta \approx \pi / 2$ since for hierarchical neutrinos $m_{3} \gg m_{2}$. An interesting special limiting case is provided by the choice $\theta=\pi / 2$, which corresponds to an R-matrix, with an off-diagonal structure,

$$
R=\left(\begin{array}{cc}
0 & 1 \\
-1 & 0
\end{array}\right)
$$

In this limiting case Eq41 shows that the vector $\underline{v_{B}}$ is exactly orthogonal to $v_{A}$. This example was discussed in the last section where it was shown to lead to Eq $\overline{35}$, where the dominant right-handed neutrino dominates the see-saw mechanism by a factor of 5 according to Eq 36. For small deviations from $\theta=\pi / 2$, Eq41 shows that the vector $\underline{v_{B}}$ is approximately orthogonal to $\underline{v_{A}}$, and as the angle $\theta$ is decreased, the vectors $\underline{v_{B}}$ and $\underline{v_{A}}$ become less orthogonal.

There is a precise correlation between the angle between the two vectors $v_{A}$ and $\underline{v_{B}}$ and the degree of dominance, parameterized by the angle $\theta$. To see this we first write Eq41 in a more compact form as,

$$
\begin{aligned}
& \underline{v_{A}}=c \underline{m}_{2}^{1 / 2}+s \underline{m}_{3}^{1 / 2} \\
& \underline{v_{B}}=-s \underline{m}_{2}^{1 / 2}+c \underline{m}_{3}^{1 / 2}
\end{aligned}
$$


where $\underline{m}_{j}^{1 / 2}$ is defined by comparing Eq43 to Eq41] as

$$
\underline{m}_{j}^{1 / 2}=\left(V_{M N S}^{2 \times 2}\right)_{i j} m_{j}^{1 / 2}
$$

i.e. the $\underline{m}_{j}^{1 / 2}$ is the $j$-th column of $V_{M N S}^{2 \times 2}$ times $m_{j}^{1 / 2}$. We see that $\underline{m}_{i}^{1 / 2} \cdot \underline{m}_{j}^{1 / 2}=\delta_{i j} m_{i}$, and $\left|\underline{m}_{2}^{1 / 2} \times \underline{m}_{3}^{1 / 2}\right|^{2}=m_{2} m_{3}$. The angle $\theta_{A B}$ between the two vectors $\underline{v_{A}}$ and $\underline{v_{B}}$ is then given by,

$$
\cos \theta_{A B}=\frac{\left(m_{3}-m_{2}\right) \sin 2 \theta}{2\left|\underline{v_{A}}\right|\left|\underline{v_{B}}\right|}
$$

where the magnitudes of the vectors is given by,

$$
\left|\underline{v_{A}}\right|^{2}=c^{2} m_{2}+s^{2} m_{3}, \quad\left|\underline{v_{B}}\right|^{2}=s^{2} m_{2}+c^{2} m_{3} .
$$

From Eqs 45 46 it is seen that the angle $\theta$ simultaneously parameterizes the angle between the two column vectors and their ratio of magnitudes which quantifies the precise degree of dominance. From Eqs 4546 it is seen that when $\theta \approx \pi / 2$, then $\theta_{A B} \approx \pi / 2$ and $\left|\underline{v_{A}}\right|^{2} /\left|\underline{v_{B}}\right|^{2} \approx m_{3} / m_{2} \approx 5$, corresponding to $\left|\underline{v_{A}}\right|^{2} \gg\left|\underline{v_{B}}\right|^{2}$ as in Eq $\underline{34}$. Once an angle $\theta$ and the right-handed neutrino masses have been chosen, and the vectors $v_{B}$ and $\underline{v_{A}}$ thereby specified, we can invert Eq43, to express the neutrino mass eigenstates in terms of the different see-saw contributions,

$$
\begin{aligned}
& \underline{m}_{2}^{1 / 2}=c \underline{v_{A}}-s \underline{v_{B}} \\
& \underline{m}_{3}^{1 / 2}=s \underline{v_{A}}+c \underline{v_{B}} .
\end{aligned}
$$

With values of $\theta \approx \pi / 2$, corresponding to single right-handed neutrino dominance, Eq47 clearly shows that the mass eigenstate $m_{3}$ mainly results from the see-saw contribution of the right-handed neutrino of mass $M_{A}$, and the mass eigenstate $m_{2}$ mainly results from the see-saw contribution of the right-handed neutrino of mass $M_{B}$. However Eq47 should be interpreted with care since it is only meaningful once Eq41 has first been used.

It is also observed from Eqs 4546 that when $\theta \approx 0$, then $\theta_{A B} \approx \pi / 2$ and $\left|\underline{v_{A}}\right|^{2} /\left|\underline{v_{B}}\right|^{2} \approx$ $m_{2} / m_{3} \approx 1 / 5$ corresponding to $\left|\underline{v_{B}}\right|^{2} \gg\left|\underline{v_{A}}\right|^{2}$. This corresponds to another type of dominance in which the heavier right-handed neutrino of mass $M_{B}$ dominates the seesaw mechanism. So far we have been assuming that the lighter right-handed neutrino of mass $M_{A}$ dominates the see-saw mechanism, but now we see that there is an alternative case in which the heavier right-handed neutrino of mass $M_{B}$ is the dominant one, and in this case we would find that the dominance of the right-handed neutrino of mass $M_{B}$ is achieved for $\theta \approx 0$, and then the $\mathrm{R}$-matrix is the unit matrix,

$$
R=\left(\begin{array}{ll}
1 & 0 \\
0 & 1
\end{array}\right)
$$


The dominance approximation is thus seen to be valid over a large range of angles $\theta$ centered on either zero or $\pi / 2$, corresponding to a large range of angles $\theta_{A B}$ in the range $\pi / 4$ to $\pi / 2$. Of course there is no precise value of $\theta$ at which the dominance approximation breaks down, and the parametrization shows that there is a continuum of theories which interpolate between those which have dominance of one right-handed neutrino and those which do not, in varying degrees. This analysis shows that the idea of single right-handed neutrino dominance is quite generic and it is quite likely to be relevant to some approximation in practice.

The above discussion illustrates that there are two types of dominance, one in which the lighter right-handed neutrino dominates, corresponding to an R-matrix with $\theta \approx \pi / 2$ like Eq42, and one in which the heavier right-handed neutrino dominates, corresponding to an R-matrix with $\theta \approx 0$ like Eq48. In practice, in dealing with the second type of dominance, it is convenient to continue to identify the heavier dominant right-handed neutrino by the label $A$ and rewriting Eq21 in this case as:

$$
M_{R R}=\left(\begin{array}{cc}
M_{B} & 0 \\
0 & M_{A}
\end{array}\right), \quad m_{L R}^{\nu}=\left(\begin{array}{cc}
B_{2} & A_{2} \\
B_{3} & A_{3}
\end{array}\right)
$$

where here $M_{B}<M_{A}$. Thus, when the heavier right-handed neutrino dominates, we shall perform a trivial relabelling $A \leftrightarrow B$ so that without loss of generality the righthanded neutrino of mass $M_{A}$ always dominates. Clearly in this second case, using Eq 49, all the results in this section from Eq 39 onwards follow as before but with a trivial relabelling $A \leftrightarrow B$. We emphasize again that the advantage of dominance is that the determinant of the neutrino mass matrix is naturally small, and also that the mixing angle is independent of the neutrino mass hierarchy, both features following from the fact that with $\theta \approx \pi / 2 \underline{v}_{A} \approx \underline{m}_{3}^{1 / 2}$ (the same result being also true for the case or $\theta \approx 0$ after relabelling $A \leftrightarrow \overline{B) \text {. }}$

\subsection{Three family SD in the see-saw flavour basis}

It is straightforward to extend two family SD in the see-saw flavour basis to the case of three families, Eq21] becomes,

$$
M_{R R}=\left(\begin{array}{ccc}
M_{A} & 0 & 0 \\
0 & M_{B} & 0 \\
0 & 0 & M_{C}
\end{array}\right), \quad m_{L R}^{\nu}=\left(\begin{array}{ccc}
A_{1} & B_{1} & C_{1} \\
A_{2} & B_{2} & C_{2} \\
A_{3} & B_{3} & C_{3}
\end{array}\right)
$$

We extend the column vector notation introduced previously,

$$
\underline{v_{A}}=\left(\begin{array}{c}
A_{1} \\
A_{2} \\
A_{3}
\end{array}\right) M_{A}^{-1 / 2}, \quad \underline{v_{B}}=\left(\begin{array}{c}
B_{1} \\
B_{2} \\
B_{3}
\end{array}\right) M_{B}^{-1 / 2}, \quad \underline{v_{C}}=\left(\begin{array}{c}
C_{1} \\
C_{2} \\
C_{3}
\end{array}\right) M_{C}^{-1 / 2}
$$


Then the see-saw formula in Eq10 $m_{L L}^{\nu}=m_{L R}^{\nu} M_{R R}^{-1} m_{L R}^{\nu}{ }^{T}$ gives:

$$
m_{L L}^{\nu}=\underline{v_{A}}{\underline{v_{A}}}^{T}+\underline{v_{B}}{\underline{v_{B}}}^{T}+\underline{v_{C}}{\underline{v_{C}}}^{T}
$$

We assume the dominance condition

$$
\frac{\left|A_{i} A_{j}\right|}{M_{A}} \gg \frac{\left|B_{i} B_{j}\right|}{M_{B}} \gg \frac{\left|C_{i} C_{j}\right|}{M_{C}} .
$$

where $i, j=1, \ldots 3$. In vector notation this implies:

$$
\left|\underline{v_{A}}\right|^{2} \gg\left|\underline{v_{B}}\right|^{2} \gg\left|\underline{v_{C}}\right|^{2} \text {. }
$$

We also assume:

$$
\left|A_{1}\right| \ll\left|A_{2,3}\right| \text {. }
$$

Then approximate results for the masses and mixings are given by [8], writing $A_{\alpha}=$ $\left|A_{\alpha}\right| e^{i \phi_{A_{1}}}, B_{\alpha}=\left|B_{\alpha}\right| e^{i \phi_{B_{1}}}, C_{\alpha}=\left|C_{\alpha}\right| e^{i \phi_{C_{1}}}$ :

$$
\begin{aligned}
\tan \theta_{23} & \approx \frac{\left|A_{2}\right|}{\left|A_{3}\right|}, \\
\tan \theta_{12} & \approx \frac{\left|B_{1}\right|}{c_{23}\left|B_{2}\right| \cos \tilde{\phi}_{2}-s_{23}\left|B_{3}\right| \cos \tilde{\phi}_{3}}, \\
\theta_{13} & \approx e^{i\left(\tilde{\phi}+\phi_{B_{1}}-\phi_{A_{2}}\right)} \frac{\left|B_{1}\right|\left(A_{2}^{*} B_{2}+A_{3}^{*} B_{3}\right)}{\left[\left|A_{2}\right|^{2}+\left|A_{3}\right|^{2}\right]^{3 / 2}} \frac{M_{A}}{M_{B}}+\frac{e^{i\left(\tilde{\phi}+\phi_{A_{1}}-\phi_{A_{2}}\right)}\left|A_{1}\right|}{\sqrt{\left|A_{2}\right|^{2}+\left|A_{3}\right|^{2}}}
\end{aligned}
$$

and for the masses

$$
\begin{aligned}
& m_{3} \approx \frac{\left(\left|A_{2}\right|^{2}+\left|A_{3}\right|^{2}\right) v^{2}}{M_{A}}, \\
& m_{2} \approx \frac{\left|B_{1}\right|^{2} v^{2}}{s_{12}^{2} M_{B}}, \\
& m_{1} \approx \mathcal{O}\left(|C|^{2} v^{2} / M_{C}\right) .
\end{aligned}
$$

The MNS phase $\delta$ is fixed by the requirement that we have already imposed in Eq. (56b) that $\theta_{12}$ is real,

$$
c_{23}\left|B_{2}\right| \sin \tilde{\phi}_{2} \approx s_{23}\left|B_{3}\right| \sin \tilde{\phi}_{3}
$$

where

$$
\begin{aligned}
& \tilde{\phi}_{2} \equiv \phi_{B_{2}}-\phi_{B_{1}}-\tilde{\phi}+\delta \\
& \tilde{\phi}_{3} \equiv \phi_{B_{3}}-\phi_{B_{1}}+\phi_{A_{2}}-\phi_{A_{3}}-\tilde{\phi}+\delta .
\end{aligned}
$$


The phase $\tilde{\phi}$ is fixed by the requirement (not yet imposed in Eq. (56c)) that the angle $\theta_{13}$ is real. In general this condition is rather complicated since the expression for $\theta_{13}$ is a sum of two terms. However if, for example, $A_{1}=0$ then $\tilde{\phi}$ is fixed by:

$$
\tilde{\phi} \approx \phi_{A_{2}}-\phi_{B_{1}}-\eta
$$

where

$$
\eta=\arg \left(A_{2}^{*} B_{2}+A_{3}^{*} B_{3}\right) .
$$

Eq. (61) may be expressed as

$$
\tan \eta \approx \frac{\left|B_{2}\right| s_{23} s_{2}+\left|B_{3}\right| c_{23} s_{3}}{\left|B_{2}\right| s_{23} c_{2}+\left|B_{3}\right| c_{23} c_{3}}
$$

Inserting $\tilde{\phi}$ in Eq. (60) into Eqs. (158), (159), we obtain a relation which can be expressed as

$$
\tan (\eta+\delta) \approx \frac{\left|B_{2}\right| c_{23} s_{2}-\left|B_{3}\right| s_{23} s_{3}}{-\left|B_{2}\right| c_{23} c_{2}+\left|B_{3}\right| s_{23} c_{3}}
$$

In Eqs. (62), (63) we have written $s_{i}=\sin \eta_{i}, c_{i}=\cos \eta_{i}$, where we have defined

$$
\eta_{2} \equiv \phi_{B_{2}}-\phi_{A_{2}}, \quad \eta_{3} \equiv \phi_{B_{3}}-\phi_{A_{3}}
$$

which are invariant under a charged lepton phase transformation. The reason why the seesaw parameters only involve two invariant phases rather than the usual six, is due to the two right-handed neutrino assumption, which removes three phases, together with the further assumption (in this case) of $A_{1}=0$, which removes another phase.

\subsection{Three family SD and the $R$ matrix}

We now discuss the R-matrix for this case. From Eq[15] we have for the two right-handed neutrino model, dropping primes and assuming $M_{A}<M_{B}<M_{C}$ :

$$
m_{L R}^{\nu}\left(\begin{array}{ccc}
M_{A} & 0 & 0 \\
0 & M_{B} & 0 \\
0 & 0 & M_{C}
\end{array}\right)^{-1 / 2}=V^{M N S}\left(\begin{array}{ccc}
m_{1} & 0 & 0 \\
0 & m_{2} & 0 \\
0 & 0 & m_{3}
\end{array}\right)^{1 / 2} R^{T} .
$$

Eq $[65$ yields the following expressions for the column vectors introduced in Eqs 51

$$
\left(\underline{v_{A}} \quad \underline{v_{B}} \quad \underline{v_{C}}\right)=\left(\begin{array}{lll}
\underline{m}_{1}^{1 / 2} & \underline{m}_{2}^{1 / 2} & \underline{m}_{3}^{1 / 2}
\end{array}\right) R^{T}
$$

where column vectors $\underline{m}_{j}^{1 / 2}$ are defined as:

$$
\underline{m}_{j}^{1 / 2}=V_{i j}^{M N S} m_{j}^{1 / 2}
$$


i.e. the column vector $\underline{m}_{j}^{1 / 2}$ is equal to the $j$-th column of $V^{M N S}$ times $m_{j}^{1 / 2}$.

The MNS matrix is given by,

$$
V^{M N S}=\left(\begin{array}{ccc}
c_{12} c_{13} & s_{12} c_{13} & s_{13} e^{-i \delta} \\
-s_{12} c_{23}-c_{12} s_{23} s_{13} e^{i \delta} & c_{12} c_{23}-s_{12} s_{23} s_{13} e^{i \delta} & s_{23} c_{13} \\
s_{12} s_{23}-c_{12} c_{23} s_{13} e^{i \delta} & -c_{12} s_{23}-s_{12} c_{23} s_{13} e^{i \delta} & c_{23} c_{13}
\end{array}\right) P_{0}
$$

where

$$
P_{0}=\left(\begin{array}{ccc}
e^{i \beta_{1}} & 0 & 0 \\
0 & e^{i \beta_{2}} & 0 \\
0 & 0 & 1
\end{array}\right) .
$$

The $R$ matrix is a complex orthogonal $3 \times 3$ matrix which can be parameterized in terms of three complex angles $\theta_{i}$ as $R=\operatorname{diag}( \pm 1, \pm 1, \pm 1) R_{1} R_{2} R_{3}$ where $R_{i}^{T}$ take the form of Eq40.

$$
\begin{aligned}
& R_{1}^{T}=\left(\begin{array}{ccc}
1 & 0 & 0 \\
0 & c_{1} & -s_{1} \\
0 & s_{1} & c_{1}
\end{array}\right), R_{2}^{T}=\left(\begin{array}{ccc}
c_{2} & 0 & -s_{2} \\
0 & 1 & 0 \\
s_{2} & 0 & c_{2}
\end{array}\right), R_{3}^{T}=\left(\begin{array}{ccc}
c_{3} & -s_{3} & 0 \\
s_{3} & c_{3} & 0 \\
0 & 0 & 1
\end{array}\right) . \\
& R_{3}^{T} R_{2}^{T} R_{1}^{T}=\left(\begin{array}{ccc}
c_{2} c_{3} & -c_{1} s_{3}-s_{1} s_{2} c_{3} & s_{1} s_{3}-c_{1} s_{2} c_{3} \\
c_{2} s_{3} & c_{1} c_{3}-s_{1} s_{2} s_{3} & -s_{1} c_{3}-c_{1} s_{2} s_{3} \\
s_{2} & s_{1} c_{2} & c_{1} c_{2}
\end{array}\right)
\end{aligned}
$$

where we have written $s_{i}=\sin \theta_{i}, c_{i}=\cos \theta_{i}$.

Although the $R$ matrix is rather complicated, it is clear from Eq [66 that SD occurs for values of angles $\theta_{i}$ which correspond to the following approximate forms for the moduli of the elements of $R^{T}$ :

$$
\begin{aligned}
& \left|R_{A B C}^{T}\right| \approx\left(\begin{array}{lll}
0 & 0 & 1 \\
0 & 1 & 0 \\
1 & 0 & 0
\end{array}\right) \Rightarrow\left(\begin{array}{lll}
\underline{v_{A}} & \underline{v_{B}} & \underline{v_{C}}
\end{array}\right) \approx\left(\begin{array}{lll}
\underline{m}_{3}^{1 / 2} & \underline{m}_{2}^{1 / 2} & \underline{m}_{1}^{1 / 2}
\end{array}\right) \\
& \left|R_{A C B}^{T}\right| \approx\left(\begin{array}{lll}
0 & 1 & 0 \\
0 & 0 & 1 \\
1 & 0 & 0
\end{array}\right) \Rightarrow\left(\begin{array}{lll}
\underline{v_{A}} \underline{v_{C}} & \underline{v_{B}}
\end{array}\right) \approx\left(\begin{array}{lll}
\underline{m}_{3}^{1 / 2} & \underline{m}_{1}^{1 / 2} & \underline{m}_{2}^{1 / 2}
\end{array}\right) \\
& \left|R_{B A C}^{T}\right| \approx\left(\begin{array}{lll}
0 & 0 & 1 \\
1 & 0 & 0 \\
0 & 1 & 0
\end{array}\right) \Rightarrow\left(\begin{array}{lll}
\underline{v_{B}} & \underline{v_{A}} & \underline{v_{C}}
\end{array}\right) \approx\left(\begin{array}{lll}
\underline{m}_{2}^{1 / 2} & \underline{m}_{3}^{1 / 2} & \underline{m}_{1}^{1 / 2}
\end{array}\right) \\
& \left|R_{B C A}^{T}\right| \approx\left(\begin{array}{lll}
0 & 1 & 0 \\
1 & 0 & 0 \\
0 & 0 & 1
\end{array}\right) \Rightarrow\left(\begin{array}{lll}
\underline{v_{B}} & \underline{v_{C}} & \underline{v_{A}}
\end{array}\right) \approx\left(\begin{array}{lll}
\underline{m}_{2}^{1 / 2} & \underline{\underline{m}}_{1}^{1 / 2} & \underline{m}_{3}^{1 / 2}
\end{array}\right)
\end{aligned}
$$




$$
\begin{gathered}
\left|R_{C A B}^{T}\right| \approx\left(\begin{array}{lll}
1 & 0 & 0 \\
0 & 0 & 1 \\
0 & 1 & 0
\end{array}\right) \Rightarrow\left(\begin{array}{lll}
\underline{v_{C}} & \underline{v_{A}} & \underline{v_{B}}
\end{array}\right) \approx\left(\begin{array}{lll}
\underline{m}_{1}^{1 / 2} & \underline{m}_{3}^{1 / 2} & \underline{m}_{2}^{1 / 2}
\end{array}\right) \\
\left|R_{C B A}^{T}\right| \approx\left(\begin{array}{lll}
1 & 0 & 0 \\
0 & 1 & 0 \\
0 & 0 & 1
\end{array}\right) \Rightarrow\left(\begin{array}{lll}
\underline{v_{C}} & \underline{v_{B}} & \underline{v_{A}}
\end{array}\right) \approx\left(\begin{array}{lll}
\underline{m}_{1}^{1 / 2} & \underline{m}_{2}^{1 / 2} & \underline{m}_{3}^{1 / 2}
\end{array}\right)
\end{gathered}
$$

As discussed in the previous section, without loss of generality we have assumed that the dominant right-handed neutrino is labelled by $A$, the leading subdominant righthanded neutrino is labelled by $B$, and the subsubdominant right-handed neutrino is labelled by $C$, and we have relabelled the right-handed neutrinos where appropriate according to this convention. The possible forms of the neutrino Dirac mass matrix $m_{L R}^{\nu}$ corresponding to the above types of SD are then given by

$$
\begin{aligned}
& m_{L R}^{\nu}=(A, B, C) \quad \text { or } \quad m_{L R}^{\nu}=(A, C, B) \text { for } M_{1}=M_{A} \text {, } \\
& m_{L R}^{\nu}=(B, A, C) \quad \text { or } \quad m_{L R}^{\nu}=(B, C, A) \text { for } M_{1}=M_{B} \text {, } \\
& m_{L R}^{\nu}=(C, A, B) \text { or } \quad m_{L R}^{\nu}=(C, B, A) \text { for } M_{1}=M_{C} \text {, }
\end{aligned}
$$

where we have ordered the columns in each case according to $M_{R R}=\operatorname{diag}\left(M_{1}, M_{2}, M_{3}\right)$ where $M_{1}<M_{2}<M_{3}$, consistent with Eq 65 .

Clearly the different types of SD correspond to the moduli of the $R$ matrix elements taking values close to either zero or unity, so that each of the vectors $\underline{v_{A}}, \underline{v_{B}}, \underline{v_{C}}$ is approximately equal to a particular vector $\underline{m}_{i}^{1 / 2}$. Considering the modular surfaces of $\sin \theta_{i}$ and $\cos \theta_{i}$, this corresponds to the angles $\theta_{i}$ being approximately real and taking values close to either zero or $\pi / 2$, which is a generalization of the situation in the two family example discussed previously. Note that SD therefore implies that the $R$ matrix is approximately real. Since there has been some recent interest in the case of the real $R$ matrix in the context of flavour-dependent leptogenesis, we shall return to this point later in Section 4

\subsection{SD in the two right-handed neutrino approximation}

In this subsection we consider the two right-handed neutrino limit of SD. We shall suppose that we have SD but not exact tri-bimaximal mixing. In this case $R$ takes the approximate the forms discussed in the previous section. For definiteness we will consider the type of SD corresponding to $R$ being close to the unit matrix. The other kinds of SD are discussed in Appendix $\mathrm{A}$

The two right-handed neutrino approximation corresponds to the limit in which the right-handed neutrino labelled by $C$ decouples from the see-saw mechanism, where this limit also corresponds to $m_{1}=0$. In this limit of SD we shall see that the models reduces 
to the two right-handed neutrino model with SD introduced in [8]. For example, let us consider the case of $R$ being approximately equal to the unit matrix, corresponding to the type of SD given in Eq 77. In the $C$ decoupling limit this corresponds to:

$$
\left(\begin{array}{lll}
\underline{0} & \underline{v_{B}} & \underline{v_{A}}
\end{array}\right)=\left(\begin{array}{lll}
\underline{0} & \underline{m}_{2}^{1 / 2} & \underline{m}_{3}^{1 / 2}
\end{array}\right) R_{0 B A}^{T} .
$$

This limit corresponds to $s_{2}=s_{3}=0$, with only $s_{1} \neq 0$, giving:

$$
R_{0 B A}^{T}=\left(R_{3}^{T}\right)_{s_{3}=0}\left(R_{2}^{T}\right)_{s_{2}=0} R_{1}^{T}=\left(\begin{array}{ccc}
1 & 0 & 0 \\
0 & c_{1} & -s_{1} \\
0 & s_{1} & c_{1}
\end{array}\right) .
$$

This results in:

$$
\begin{aligned}
& \underline{v_{B}}=c_{1} \underline{m}_{2}^{1 / 2}+s_{1} \underline{m}_{3}^{1 / 2} \\
& \underline{v_{A}}=-s_{1} \underline{m}_{2}^{1 / 2}+c_{1} \underline{m}_{3}^{1 / 2}
\end{aligned}
$$

similar to Eq43 in the two family model, except that here the vectors have three components. SD here corresponds to a small angle $\theta_{1} \approx 0$ (for both real and imaginary components). A zero value $\theta_{1}=0$ implies that $A_{1} \propto \theta_{13}$, as discussed. However a non-zero angle $\theta_{1}$ allows for example a zero value of $A_{1}=0$ consistent with a non-zero value of $\theta_{13}$. For example $A_{1}=0$ implies from Eq 83 ,

$$
\tan \theta_{1} \approx\left(\frac{m_{3}}{m_{2}}\right)^{1 / 2} e^{-i\left(\delta+\beta_{2}\right)} \frac{\tan \theta_{13}}{s_{12}}
$$

This result shows that, with a texture zero $A_{1}=0$, small $\theta_{13}$ implies also small $\theta_{1}$. This is a remarkable result: in general having a small value of $A_{1}$ combined with small $\theta_{13}$ in the two right-handed neutrino limit implies also small (but non-zero in general) $\theta_{1}$, corresponding to SD. In the two right-handed neutrino limit it is impossible to have a texture zero $A_{1}$ without $\mathrm{SD}$.

A similar analysis follows for the other types of SD, where the right-handed neutrino labelled by $C$ in these cases can be decoupled in a similar way. In each case it is necessary to allow the remaining dominant and subdominant right-handed neutrinos to mix, in order to allow for the most general kind of SD, and we identify the single remaining mixing angle in each case. The other cases are discussed in Appendix A.

The above discussion and Appendix $\mathrm{A}$ shows how an effective two right-handed neutrino model arises as a limiting case of the three right-handed neutrino model in which the right-handed neutrino labelled by $C$ is decoupled. In this decoupling limit the remaining two right-handed neutrino system is parameterized in each case by a single non-trivial complex angle, where the nature of the angle and the values of the other fixed angles of the $R$ matrix depend on the type of three right-handed neutrino 
SD. In particular the limiting cases all led to relations similar to Eq43 which is repeated below:

$$
\begin{aligned}
& \underline{v_{A}}=c \underline{m}_{2}^{1 / 2}+s \underline{m}_{3}^{1 / 2} \\
& \underline{v_{B}}=-s \underline{m}_{2}^{1 / 2}+c \underline{m}_{3}^{1 / 2}
\end{aligned}
$$

where the main difference is that the vectors here have three components. For each type of SD it is straightforward to relate the angle $\theta$ to either $\theta_{1}$ or $\theta_{2}$ using the results given in Eqs 83117120123126, 129, The following discussion will be based on the angle $\theta$ defined in Eq,85, assuming that this identification has been made.

Eq 85] again leads to a similar geometrical relation between the $R$ matrix angle $\theta$ and the angle between the two vectors $v_{A}$ and $v_{B}$ as in Eq45, where the magnitudes of the two vectors is as in Eq46. These results follow from the unitarity of $V_{C K M}$ (since recall that $\underline{m}_{i}^{1 / 2}$ is proportional to the $i-t h$ column of $\left.V_{C K M}\right)$ which gives:

$$
\left\langle\underline{m}_{i}^{1 / 2} \mid \underline{m}_{j}^{1 / 2}\right\rangle=\delta_{i j} m_{j}
$$

and hence:

$$
\begin{aligned}
& \left\langle\underline{v_{A}} \mid \underline{v_{B}}\right\rangle=-c^{*} s m_{2}+s^{*} c m_{3} \\
& \left\langle\underline{v_{A}} \mid \underline{v_{A}}\right\rangle=c^{*} c m_{2}+s^{*} s m_{3} \\
& \left\langle\underline{v_{B}} \mid \underline{v_{B}}\right\rangle=s^{*} s m_{2}+c^{*} c m_{3} .
\end{aligned}
$$

In the case of tri-bimaximal mixing $s=0$ or $c=0$ and hence $\left\langle\underline{v_{A}} \mid \underline{v_{B}}\right\rangle=0$, i.e. orthogonality of the dominant and subdominant columns of the Yukawa matrix, as in Eq191. However, as the previous discussion shows, away from the tri-bimaximal limit these limits are in general too strong, and so we must in general consider $s, c \neq 0$, with SD corresponding to either $s \approx 0$, or $c \approx 0$, which implies the $R$ matrix angle $\theta$ takes approximately real values close to zero or $\pi / 2$. We also remark that it is trivial to generalize the result in Eq84 to all the other types of SD. In other words a texture zero $A_{1}=0$ directly implies SD, for each of the types of SD.

It is possible to regard the two right-handed neutrino model as a complete model in its own right, not as a limiting case of a three right-handed neutrino model. This is not so well motivated as the limiting cases discussed here. However in such a case one may take Eq 85] as the starting point for the exploration of the parameter space. This has been discussed fully elsewhere [16], so we shall not pursue this point further here. However the results in this subsection should be useful in relating a three right-handed neutrino analysis to the two right-handed neutrino limit, and in particular to the SD regions of parameter space of this limit. 


\section{Applications of basis independent SD}

In this section we first discuss the application of these new ideas to flavour models, then discuss the implications for approaches based on the $R$ matrix, including flavourdependent leptogenesis which has recently been studied in the literature.

\subsection{Examples of models in the same invariant class as SD}

The usual application of SD to flavour models in the literature is in the see-saw flavour basis corresponding to diagonal mass matrices of charged leptons and right-handed neutrinos, or small perturbations away from the diagonal structures. This severely restricts the applicability of SD, and may even lead one to believe that SD is an artefact of that particular basis, or could be transformed away by going to another basis, or even that it is meaningless since all see-saw models are related to each other by a change of basis. We have shown explicitly in this paper that none of these statements is true. We have shown how the different types of SD may be formulated in a basis independent way in terms of the $R$ matrix, since, as we have also shown, each choice of $R$ matrix labels an infinite equivalence class of see-saw models related to each other by changes of lepton basis. These results open the door for new applications of SD away from the usual diagonal basis of charged leptons and right-handed neutrinos. In this subsection we illustrate the possibilities by highlighting some existing models in the literature which are now seen to be SD in disguise, i.e. are in the same invariant class as SD.

\subsubsection{Tri-bimaximal neutrino mixing and CSD: charged lepton corrections}

In this subsection, we first discuss CSD and tri-bimaximal neutrino mixing in terms of the $R$ matrix. We shall show that the $R$ matrix elements take quite precise values equal to either zero or plus or minus unity (we shall discuss how precise) in this case, which are unaffected by charged lepton corrections, according to section 2.4 in which a change of charged lepton basis leaves the $R$ matrix invariant. However the MNS matrix is subject to observable deviations from tri-bimaximal mixing due to charged lepton corrections. The lesson from this is that the charged lepton corrections can result in a change of the invariant class of see-saw model, not due to a change in $R$ but due to a change in the physical parameters.

In the notation of Eq[50, tri-bimaximal mixing [3] corresponds to the choice [10]:

$$
\begin{aligned}
\left|A_{1}\right| & =0, \\
\left|A_{2}\right| & =\left|A_{3}\right|, \\
\left|B_{1}\right| & =\left|B_{2}\right|=\left|B_{3}\right|, \\
A^{\dagger} B & =0 .
\end{aligned}
$$


This is called constrained SD (CSD) [10]. Note that there is no constraint imposed on the couplings $C_{i}$ since these describe the right-handed neutrino which is approximately decoupled from the see-saw mechanism.

In terms of the $R$ matrix SD corresponds to the special case that the $R$ matrix elements are approximately equal to zero or plus or minus unity. We now show that the accurate limit of SD, in which the elements of $R$ are zero or plus or minus unity very accurately, corresponds to CSD and tri-bimaximal mixing. We shall consider the case of the $R$ matrix approximately equal to the unit matrix (the other cases follow similarly). In this case we can write Eq[7] explicitly as:

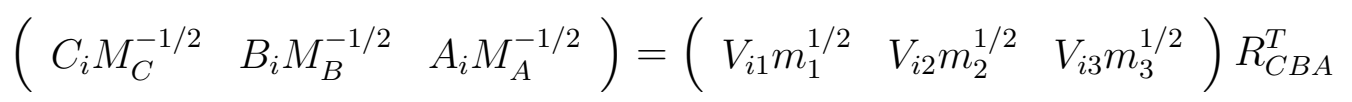

where we have written $V_{i j}=V_{i j}^{M N S}$. If we take $R_{C B A}^{T}=\operatorname{diag}(1,1,1)$ precisely, then Eq92 implies for example that $A_{i} \propto V_{i 3}$, so that $A_{1}=0$ would imply that $\theta_{13}=0$ (c.f. the general case from Eq $56 \mathrm{c}$ where $\theta_{13}$ involves a contribution from a term which is independent of $\left.A_{1}\right)$. We further note that for $R_{C B A}^{T}=\operatorname{diag}(1,1,1)$ and for tri-bimaximal mixing angles $\theta_{13}=0, \sin \theta_{23}=1 / \sqrt{2}, \sin \theta_{12}=1 / \sqrt{3}$, the Dirac matrix takes a very special form:

$$
\begin{aligned}
& \left(\begin{array}{l}
A_{1} \\
A_{2} \\
A_{3}
\end{array}\right) \propto\left(\begin{array}{c}
0 \\
s_{23} \\
c_{23}
\end{array}\right) \propto\left(\begin{array}{l}
0 \\
1 \\
1
\end{array}\right) \\
& \left(\begin{array}{c}
B_{1} \\
B_{2} \\
B_{3}
\end{array}\right) \propto\left(\begin{array}{c}
s_{12} \\
c_{12} c_{23} \\
-c_{12} s_{23}
\end{array}\right) \propto\left(\begin{array}{c}
1 \\
1 \\
-1
\end{array}\right),
\end{aligned}
$$

ignoring the irrelevant couplings $C_{i}$. These satisfy the CSD conditions for the Yukawa couplings discussed in Eqs 88,91 [10]. We conclude that with $R$ precisely equal to the unit matrix tri-bimaximal mixing implies and is implied by CSD. Of course this is not the only way to achieve tri-bimaximal mixing, which could be achieved via any other choice of $R$-matrix, corresponding to other choices of Yukawa couplings, but this choice of Yukawa couplings appears to be the simplest, and could arise for example from vacuum alignment in flavour models [10, 12. Indeed the simplicity of the Yukawa couplings in this case provides a powerful motivation for SD. Similar forms of the Yukawa matrices of the CSD form for tri-bimaximal mixing emerge from the other types of SD in Eqs:77, 73 when the $R$ matrices take the exact forms shown there (with the elements being precisely $0, \pm 1)$ rather than just the approximate forms.

In realistic models [10, 12] it is typically the case that CSD arises through vacuum alignment in the some theory basis, in which the charged lepton mass matrix is not precisely diagonal, resulting in charged lepton corrections to tri-bimaximal mixing. In the theory basis there is, to good approximation, tri-bimaximal neutrino mixing, and 
the neutrino Dirac mass matrix is parameterized in terms of a unit $R$ matrix (or one of the other exact forms in Eqs 72, (77) as we have just seen. However, if, in some basis, the $R$ matrix is equal to the unit matrix, for example, then this will be true in all bases, as we showed in section 2.4. In the presence of charged lepton corrections the MNS matrix will deviate from the tri-bimaximal form, but the $R$ matrix will remain equal to the unit matrix. In going from the theory basis to the see-saw flavour basis in which the charged lepton mass matrix is diagonal, both sides of Eq17 must be left-multiplied by a matrix $V_{E_{L}}$ which diagonalizes the charged leptons, resulting in $V_{M N S}^{\prime}$ appearing on the right-hand side which is not of the precise tri-bimaximal form, even though $R$ is precisely equal to the unit matrix in both the original basis and the primed basis. Interestingly, the neutrino mass matrix in the primed basis will retain the property that its columns are proportional to the columns of the MNS matrix, albeit that the MNS matrix is not precisely of the tri-bimaximal form.

We have seen that tri-bimaximal neutrino mixing from CSD corresponds to the $R$ matrix taking one of the forms in Eqs, 72 , 77 rather precisely. One may ask how accurately should these forms be achieved in realistic models? In practice, tri-bimaximal neutrino mixing relies on the conditions in Eqs 88, 91 being satisfied which leads to tri-bimaximal mixing up to corrections of order $m_{2} / m_{3}$. The conditions on the couplings $C_{i}$ are more unconstrained since they only give corrections to the mixing angles of order $m_{1} / m_{3}$, which may be quite small. We have already examined the limit where the right-handed neutrino labelled by $C$ decouples and in this limit the corrections to tri-bimaximal neutrino mixing of order $m_{2} / m_{3}$ can be decribed by a single small angle $\theta$ as discussed in section 3.5. For example, in the case of $R$ being close to the unit matrix, then $R$ is described by $\theta_{2}=\theta_{3}=0$ with small values of $\theta_{1} \approx 0$ parameterizing the corrections of order $m_{2} / m_{3}$, according to Eq 83 . If we relax the decoupling of $C$ then we can also account for corrections of order $m_{1} / m_{3}$ to the $R$ matrix, described by non-zero values of $\theta_{2} \approx 0$ and $\theta_{3} \approx 0$, which corresponds to:

$$
\underline{v_{C}}=c_{3} c_{2} \underline{m}_{1}^{1 / 2}+s_{3} c_{2} \underline{m}_{2}^{1 / 2}+s_{2} \underline{m}_{3}^{1 / 2} \text {. }
$$

We conclude that the case of CSD and tri-bimaximal neutrino mixing corresponds to the $R$ matrix taking quite exactly (up to corrections of order $m_{1} / m_{3}, m_{2} / m_{3}$ ) one of the forms in Eqs 72, [77. If the forms of the $R$ matrix deviate by more that this, but still resemble those forms to some degree then we merely have SD not CSD, and exact tri-bimaximal neutrino mixing is lost. In the case of CSD, the presence of charged lepton mixing corrections will give observable corrections to tri-bimaximal mixing in the MNS matrix, resulting in testable predictions and sum rules for lepton mixing angles [10, 13, however these corrections leave the $R$ matrix unchanged from the precise forms just described. These precise forms of the $R$ matrix therefore represent the basis-independent signature of CSD and tri-bimaximal neutrino (rather than MNS) mixing which can be identified in phenomenological analyses based on the $R$ matrix. 


\subsubsection{Lepton mixing from the charged lepton sector}

We now discuss a class of models which account for lepton mixing purely as arising from the charged lepton sector. Such models have been discussed in [14], and we show here that they are in the same invariant class as SD models, i.e. are SD models in disguise. The starting point of these models is to assume that there is no mixing coming from the neutrino sector. The mass matrices are then written as:

$$
m_{L R}^{E}=\left(\begin{array}{ccc}
p & d & a \\
q & e & b \\
r & f & c
\end{array}\right), v_{u} Y_{L R}^{\nu}=\left(\begin{array}{ccc}
C_{1}^{\prime} & 0 & 0 \\
C_{2}^{\prime} & B_{2}^{\prime} & 0 \\
C_{3}^{\prime} & B_{3}^{\prime} & A_{3}^{\prime}
\end{array}\right), M_{R R} \approx\left(\begin{array}{ccc}
M_{C} & 0 & 0 \\
0 & M_{B} & 0 \\
0 & 0 & M_{A}
\end{array}\right)
$$

and the following conditions are assumed:

$$
\frac{\left|A_{3}^{\prime} A_{3}^{\prime}\right|}{M_{A}} \gg \frac{\left|B_{i}^{\prime} B_{j}^{\prime}\right|}{M_{B}} \gg \frac{\left|C_{i}^{\prime} C_{j}^{\prime}\right|}{M_{C}}
$$

which is the usual SD condition in Eq [53, and leads to $m_{L L}^{\nu} \approx \operatorname{diag}\left(m_{1}, m_{2}, m_{3}\right)$. We also assume the new conditions:

$$
\begin{aligned}
& |a|,|b|,|c| \gg|d|,|e|,|f| \gg|p|,|q|,|r| \\
& |d|,|e| \ll|f| .
\end{aligned}
$$

The charged lepton masses are given by:

$$
\begin{aligned}
& m_{\tau} \approx\left(|a|^{2}+|b|^{2}+|c|^{2}\right)^{\frac{1}{2}}, \\
& m_{\mu} \approx\left(|d|^{2}+|e|^{2}+|f|^{2}-\frac{\left|d^{*} a+e^{*} b+f^{*} c\right|^{2}}{m_{\tau}^{2}}\right)^{\frac{1}{2}}, \\
& m_{e} \approx \mathcal{O}(|p|,|q|,|r|) .
\end{aligned}
$$

In leading order in $|d| /|f|$ and $|e| /|f|$, the mixing angles are given by [14]:

$$
\begin{aligned}
\tan \left(\theta_{12}\right) & \approx \frac{|a|}{|b|} \\
\tan \left(\theta_{23}\right) & \approx \frac{s_{12}|a|+c_{12}|b|}{|c|}, \\
\tan \left(\theta_{13}\right) & \approx O\left(\frac{|e|,|d|}{|f|}\right) .
\end{aligned}
$$

According to the ISS approach, we should begin by calculating the $R$ matrix in the basis defined in Eq 96, in order to determine the invariant class $\mathcal{C}(R)$ to which this model belongs. For this purpose we shall use the results in section 2.4 and in particular 
Eq16 which is valid for a general charged lepton basis, but a diagonal right-handed neutrino mass basis. Here $V_{\nu_{L}}$ being the matrix that diagonalizes $m_{L L}^{\nu}$ in this basis, is actually equal to the unit matrix, since by construction there is no mixing coming from the neutrino sector. Thus the $R$ matrix is determined from Eq16 as:

$$
R^{T}=\operatorname{diag}\left(m_{1}, m_{2}, m_{3}\right)^{-1 / 2} v_{u} Y_{L R}^{\nu} \operatorname{diag}\left(M_{C}, M_{B}, M_{A}\right)^{-1 / 2}
$$

where $Y_{L R}^{\nu}$ is as in Eq96. By explicit multiplication, using the conditions for a neutrino mass hierarchy in Eq97, it is easy to see that $R$ is approximately equal to the unit matrix. It is also easy to see that if $Y_{L R}^{\nu}$ were taken to be diagonal, then $R$ would be exactly equal to the unit matrix. We already saw in section 3.4 that a unit $R$ matrix defines a particular invariant class of models to which SD belongs, where the dominant (subdominant) right-handed neutrino is the heaviest (intermediate) one. Therefore we conclude that the charged lepton mixing model here is in the same invariant class as SD.

We can check this result explictly by rotating the above models to the usual SD models by a change of charged lepton basis, using the symmetry $U_{L}(3) \times U_{E_{R}}(3)$. We thus perform a change of charged lepton basis, using the symmetry $U_{L}(3) \times U_{E_{R}}(3)$, which results in a change of mass matrices from the above ones in Eq 96 to the ones in the see-saw flavour basis in which the charged lepton mass matrix is diagonal, $m_{L R}^{E^{\prime}}=$ $\operatorname{diag}\left(m_{e}, m_{\mu}, m_{\tau}\right)$, given by:

$$
m_{L R}^{E^{\prime}}=V_{E_{L}} m_{L R}^{E} V_{E_{R}}^{\dagger}, \quad m_{L R}^{\nu^{\prime}}=V_{E_{L}} m_{L R}^{\nu}, \quad m_{L L}^{\nu^{\prime}}=V_{E_{L}} m_{L L}^{\nu} V_{E_{L}}^{T} .
$$

In the unprimed basis $m_{L L}^{\nu} \approx \operatorname{diag}\left(m_{1}, m_{2}, m_{3}\right)$, and by comparing Eq 8 to Eq 103 we identify:

$$
V_{E_{L}} \approx V_{M N S}^{\prime}
$$

Then using Eq,104 with Eq[103 we have,

$$
m_{L R}^{\nu^{\prime}} \approx V_{M N S}^{\prime} m_{L R}^{\nu}
$$

Using Eq105 with Eq 96, and the MNS matrix in Eq68, immediately leads to the SD form in Eq 50 of the neutrino mass matrix, satisfying the usual conditions in Eqs 53 , 55. with the right-handed neutrino mass ordering of the form in Eq 77. By a reordering of the right-handed neutrino masses in Eq96 we could similarly arrive at any of the types of SD in Eqs:72, 77 in the primed basis in which the charged lepton mass matrix is diagonal as in Eq,12.

Alternatively, we could start from one of the sequential right-handed neutrino dominance types, in the primed basis, then rotate to the unprimed basis in which the mixing is coming from the charged lepton sector. Starting from the primed basis, in which 
the charged lepton mass matrix is diagonal, rotating to the unprimed basis leads to $m_{L L}^{\nu} \approx \operatorname{diag}\left(m_{1}, m_{2}, m_{3}\right)$, and a charged lepton mass matrix given by:

$$
m_{L R}^{E} \approx V_{M N S}^{\prime \dagger} \operatorname{diag}\left(m_{e}, m_{\mu}, m_{\tau}\right) \text {. }
$$

For example, for tri-bimaximal mixing, Eq106 gives:

$$
m_{L R}^{E} \approx\left(\begin{array}{ccc}
\sqrt{\frac{2}{3}} m_{e} & -\sqrt{\frac{1}{6}} m_{\mu} & \sqrt{\frac{1}{6}} m_{\tau} \\
\sqrt{\frac{1}{3}} m_{e} & \sqrt{\frac{1}{3}} m_{\mu} & -\sqrt{\frac{1}{3}} m_{\tau} \\
0 & \sqrt{\frac{1}{2}} m_{\mu} & \sqrt{\frac{1}{2}} m_{\tau}
\end{array}\right)
$$

which is of the form in Eq96, for the case of tri-bimaximal lepton mixing.

We conclude that the class of models proposed in [14, where all the mixing arises from the charged lepton sector, are in the same invariant class as SD, where all mixing arises from the neutrino sector. The two types of model are in the same invariant class since they correspond to the same approximately unit $R$ matrix. In the basis in which there is no mixing coming from the neutrino sector, then $V_{\nu_{L}}^{\dagger}$ is equal to the unit matrix, while in the basis in which all the mixing is coming from the neutrino sector then $V_{\nu_{L}}^{\dagger}=V_{M N S}^{\prime}$, with $R$ being the same in both bases.

\subsubsection{Non-diagonal right-handed neutrino models}

We now consider an example of a see-saw model in which some of the mixing arises from the right-handed neutrino sector. Specifically we consider the flavour model of tri-bimaximal neutrino mixing based on $S U(3)$ or its discrete subgroup $\Delta(27)$ [12. We shall show that this model is in the same invariant class as CSD models, i.e. is CSD in disguise. This will also provide an example of how the $S$ matrix may be used as a short-cut to finding the $R$ matrix, and also the neutrino mass matrix itself.

In the model under consideration the neutrino mass matrices are of the leading order form:

$$
M_{R R}=\left(\begin{array}{ccc}
M_{A} & M_{A} & 0 \\
M_{A} & M_{A}+M_{B} & 0 \\
0 & 0 & M_{C}
\end{array}\right), \quad v_{u} Y_{L R}^{\nu}=\left(\begin{array}{ccc}
0 & B & C_{1} \\
A & B+A & C_{2} \\
-A & B-A & C_{3}
\end{array}\right)
$$

where $M_{A}<M_{B}<M_{C}$ and the couplings $A, B, C_{i}$ satisfy the conditions in Eq 53 , However it is not at all clear that the model corresponds to SD since the right-handed neutrino mass matrix is not diagonal. Moreover it is not clear that tri-bimaximal neutrino mixing results from Eq108 since it does not satisfy the CSD conditions in Eqs 88, 91,

However, using the $S$ matrix transformations in Eq18, with

$$
S^{-1}=\left(\begin{array}{ccc}
1 & -1 & 0 \\
0 & 1 & 0 \\
0 & 0 & 1
\end{array}\right)
$$


results in:

$$
M_{R R} \rightarrow\left(\begin{array}{ccc}
M_{A} & 0 & 0 \\
0 & M_{B} & 0 \\
0 & 0 & M_{C}
\end{array}\right), \quad v_{u} Y_{L R}^{\nu} \rightarrow\left(\begin{array}{ccc}
0 & B & C_{1} \\
A & B & C_{2} \\
-A & B & C_{3}
\end{array}\right)
$$

where the transformed mass matrices satisfy the CSD conditions in Eqs 88, 91. The transformed theory (not strictly a basis transformation since $S$ is not unitary) has the same $R$ matrix as the original theory, according to Eq20, even though the right-handed neutrino masses are different (note that in Eq110 $M_{A, B, C}$ are not the eigenvalues).

Having made this $S$ matrix transformation, we can calculate the neutrino mass matrix and the $R$ matrix in the transformed basis, since both quantities are invariant under $S$ as shown on section 2.4. In fact it is manifestly clear from Eq 110 that the transformed theory satisfies the CSD conditions and leads to tri-bimaximal neutrino mixing. The $R$ matrix may be obtained from Eq20,

$$
R^{T}=\operatorname{diag}\left(m_{1}, m_{2}, m_{3}\right)^{-1 / 2} V_{\nu_{L}} v_{u} Y_{L R}^{\nu} S^{-1} \operatorname{diag}\left(M_{A}, M_{B}, M_{C}\right)^{-1 / 2}
$$

where in this case $V_{\nu_{L}}^{\dagger}=V_{M N S}$ (ignoring small charged lepton corrections). In this case Eq111, with the tri-bimaximal MNS matrix, leads to an $R$ matrix of the form in Eq.72. We thus see that the original theory is in the same invariant class as CSD since it corresponds to the same $R$ matrix, in this case that given in Eq.72.

\subsection{SD phenomenology and the $R$ matrix}

In this paper we have formulated SD in terms of the $R$ matrix in order to show its basisindependence, using the fact that the $R$ matrix labels distinct equivalence classes of seesaw models, and each choice of $R$ matrix generates a continuously infinite class of models related to each other by basis transformations. However this identification has additional practical benefits since the $R$ matrix has been extensively used in phenomenological analyses, so it is useful to be able to identify sequential dominance with particular points in $R$ matrix parameter space. In this subsection we discuss some recent examples of this.

\subsubsection{Lepton flavour violation}

A recent phenomenological analysis of lepton flavour violation identified a particularly interesting region of parameter space in which the $R$ matrix is equal to or close to the unit matrix [17]. From our results here we see that the case that $R$ being exactly equal to the unit matrix corresponds to CSD and tri-bimaximal mixing, of the kind where the heaviest right-handed neutrino is the dominant one, and the second heaviest is the leading sub-dominant one. 


\subsubsection{Two right-handed neutrino model}

Another example of phenomenological analyses which have relied heavily on the $R$ matrix are the recent analyses of the two right-handed neutrino model [16. We have already shown how this can emerge from the three right-handed neutrino model by decoupling the right-handed neutrino labelled by $C$. Although in general the remaining two righthanded neutrinos in the analysis in [16] do not satisfy the SD condition (or strictly the single right-handed neutrino dominance condition, since such models automatically satisfy at least the SD condition that one of the right-handed neutrinos is decoupled) it is in fact satisfied in much of the parameter space considered, namely where the $R$ angle is close to zero or $\pi / 2$, how close being a matter being discussed earlier in this paper. Moreover having a particular texture zero, as is assumed over some regions of the analysis in [16], automatically implies SD, as we also saw earlier in Eq 84,

\subsubsection{Flavour-dependent leptogenesis}

One of the main phenomenological applications of the $R$ matrix is to leptogenesis. It is particularly convenient here since, for example, when it is used in the calculation of the flavour-independent asymmetry parameter $\epsilon_{1}$ it clearly shows that the MNS parameters cancel out. However recently there has been some activity related to the flavour-dependence of leptogenesis [18, and here the MNS parameters do not cancel out of the expressions for the separate flavour-dependent asymmetries $\epsilon_{\alpha}$, where $\alpha=e, \mu, \tau$. Nevertheless, the $R$ matrix has continues to be of interest in recent phenomenological analyses of flavour-dependent leptogenesis [19], with the flavour-dependent asymmetry parameter being given by:

$$
\epsilon_{\alpha}=-\frac{3}{16 \pi} \frac{M_{1}}{v_{u}^{2}} \frac{\operatorname{Im}\left(\sum_{\beta, \rho} m_{\beta}^{1 / 2} m_{\rho}^{3 / 2} U_{\alpha \beta}^{*} U_{\alpha \rho} R_{1 \beta}^{*} R_{1 \rho}^{*}\right)}{\sum_{\gamma} m_{\gamma}\left|R_{1 \gamma}\right|^{2}}
$$

where we have written $U=V_{M N S}$. Since Eq112 only involves basis invariant quantities, it is manifest that the asymmetry parameter $\epsilon_{\alpha}$ will take a unique value for all see-saw models which belong to a particular invariant class $\mathcal{C}(R)$, i.e. $\epsilon_{\alpha}$ is basis invariant, as it should be. The use of a real $R$ matrix to permit a link between leptogenesis and the MNS phases has been explored [19, since it then follows from Eq[112 that

$$
\epsilon_{\alpha} \propto \sum_{\beta} \sum_{\gamma>\beta} \sqrt{m_{\beta} m_{\gamma}}\left(m_{\gamma}-m_{\beta}\right) R_{1 \beta} R_{1 \gamma} \operatorname{Im}\left(U_{\alpha \beta}^{*} U_{\alpha \gamma}\right)
$$

which clearly shows that $\epsilon_{\alpha}$ only depends on the MNS phases for the case of $R$ real. A rather nice application of our results is that approximately real $R$ is an automatic consequence of SD, as we now discuss.

The case of $R$ being real has been identified with reality of the right-handed rotations used to diagonalize the Dirac matrix, and thus with the notion of there being no CP 
violation in the right-handed neutrino sector. However, this looks like quite a strong requirement. For example one way to achieve this would be to have an $S O(3)$ family symmetry in the right-handed neutrino sector, which is broken spontaneously by real flavon vacuum expectation values, which is a rather precise requirement and not at all generic. This leads to the question of whether there is any more natural way to guarantee having a real $R$ matrix which is better motivated? Our formulation of SD in terms of the $R$ matrix shows that the SD cases actually correspond to the $R$ matrix being approximately real. As discussed previously, this follows by considering the modular surfaces of $\sin \theta_{i}$ and $\cos \theta_{i}$, where we saw that $\mathrm{SD}$ corresponds to the $R$ angles $\theta_{i}$ being approximately real and taking values close to either zero or $\pi / 2$. Thus SD is a very nice way of motivating a real $R$ matrix, where $R$ takes values approximately as given in Eqs $72,77$.

The case of CSD and exact tri-bimaximal mixing, corresponding to the $R$ matrix taking quite precisely (rather than just approximately) one of the forms in Eqs [72]77] leads to zero leptogenesis asymmetry parameters. For example when $R$ is precisely equal to the unit matrix Eq113 shows that the asymmetry parameters are all equal to zero [19. Similarly for the other exact forms in Eqs,72, 177. Interestingly this result also applies to the case of tri-bimaximal neutrino mixing, with charged lepton corrections to tri-bimaximal mixing as discussed in [10] giving significant corrections to the total lepton mixing, resulting in deviations from tri-bimaximal lepton mixing. This might seem paradoxical since physically if there is no exact tri-bimaximal lepton mixing, then one might also expect that the asymmetry parameters are also not exactly zero. However the point is that, as already mentioned, if in some basis the $R$ matrix is equal to the unit matrix, then this will be true in all bases, as we showed in section 2.4. In the presence of charged lepton corrections the MNS matrix will deviate from the tri-bimaximal form, but the $R$ matrix will remain equal to the unit matrix, and leptogenesis will remain zero.

Does this mean that the asymmetry parameters of leptogenesis are always equal to zero for tri-bimaximal neutrino mixing arising from CSD? In practice, tri-bimaximal neutrino mixing in realistic models is achieved by using vacuum alignment for the dominant and leading sub-dominant right-handed neutrinos, such that the conditions in Eqs 88 -91 are satisfied. As already discussed, there are expected to be small deviations from these precise forms parameterized by small angles which represent corrections of order $m_{1} / m_{3}$ and $m_{2} / m_{3}$. In particular there are no conditions imposed on the couplings $C_{i}$ since the associated right-handed neutrino is assumed to play a negligible role in the see-saw mechanism and gives corrections of order $m_{1} / m_{3}$.

If the almost decoupled right-handed neutrino labelled by $C$ is the heaviest, or the intermediate mass right-handed neutrino, then it will also play no important role in leptogenesis, since the asymmetry parameters are determined up to corrections of order $m_{1} / m_{3}$ by the dominant and sub-dominant couplings $A_{i}, B_{i}$ [20]. However if the almost decoupled right-handed neutrino is the lightest $M_{1}=M_{C}$, then it is unavoidable that 
the couplings $C_{i}$ must be involved in the calculation of the asymmetry parameters, since the asymmetry parameters are given in this case by [20]:

$$
\epsilon_{\alpha}=-\frac{3 M_{1}}{16 \pi}\left\{\frac{\operatorname{Im}\left[C_{\alpha}^{*} A_{\alpha}\left(C^{\dagger} A\right)\right]}{M_{A}\left(C^{\dagger} C\right)}+\frac{\operatorname{Im}\left[C_{\alpha}^{*} B_{\alpha}\left(C^{\dagger} B\right)\right]}{M_{B}\left(C^{\dagger} C\right)}\right\}
$$

In this case, there are no constraints on the couplings $C_{i}$ from CSD and in particular $C^{\dagger} A$ and $C^{\dagger} B$ are both non-zero, in contrast to the other cases which would involve $A^{\dagger} B=0$ due to the CSD relation in Eq91. In the case that the $R$ matrix is precisely equal to the unit matrix, or one of the other related forms, then the column vectors $A, B, C$ are each associated with a column of the MNS matrix, and so we would have $C^{\dagger} A=C^{\dagger} B=0$ by unitarity, giving zero values of the asymmetry parameters in this case, in agreement with the general argument previously for the case of $R$ being equal to the unit matrix. However, since the couplings $C_{i}$ are unconstrained, this implies that the $R$ matrix is not precisely equal to the unit matrix, but has important corrections parameterized by non-zero values of the $R$ angles $\theta_{i}$ as discussed in Eq 95. In [20] a particular example of this type was studied in detail.

\section{Conclusion}

We have proposed an ISS approach to model building, based on the observation that see-saw models of neutrino mass and mixing fall into basis invariant classes labelled by the Casas-Ibarra $R$-matrix. We have proved that the $R$-matrix is invariant not just under basis transformations but also non-unitary right-handed neutrino transformations $S$. According to the ISS approach, given any see-saw model in some particular basis one may determine the invariant $R$ matrix and hence the invariant class to which that model belongs. The formulation of see-saw models in terms of invariant classes puts them on a firmer theoretical footing, and allows different see-saw models in the same class to be related more easily, while their relation to the $R$-matrix makes them more easily identifiable in phenomenological studies. We have also presented an ISS mass formula in Eq13, which may prove useful in model building.

We have systematically studied SD as a prime example of the ISS approach. We considered a simple two family example, before proceeding to the three family case. A very convenient vector notation was introduced in which the invariant combination $v_{u} Y_{L R}^{\nu} M_{R R}^{-1 / 2}$ on the left-hand side of Eq[15] was expressed in terms of three "Yukawa vectors" consisting of the columns of the Yukawa matrix normalized by the inverse square roots of right-handed neutrino masses as in Eq151. These three "Yukawa vectors" are then related to the "MNS vectors", consisting of columns of the MNS matrix normalized by square roots of neutrino masses, as in Eq 66. This gives a very nice physical interpretation of the $R$ matrix, as that matrix which controls the misalignment of the "Yukawa vectors" and the "MNS vectors". SD corresponds to the "Yukawa vectors" and 
"MNS vectors" being approximately aligned, up to permutations. CSD corresponds to the "Yukawa vectors" and "MNS vectors" being very accurately aligned, up to permutations. This interpretation can be extended to any right-handed neutrino or charged lepton basis providing one uses Eq[17, since the left-hand side is invariant under righthanded neutrino transformations, and on the right-hand side MNS mixing is replaced by neutrino mixing.

We have thus shown that SD models form basis invariant classes in which the $R$ matrix is approximately related to a permutation of the unit matrix, and quite accurately so in the case of CSD and tri-bimaximal neutrino mixing. We also discussed the two right-handed neutrino limit of SD. The $R$ matrix thus provides a beautiful basis invariant formulation of SD and CSD. This means that SD is physically meaningful, e.g. not all classes of see-saw models correspond to SD, and also SD cannot be transformed away by a change of basis, since the $R$ matrix is invariant under a basis change. The basis independence of SD also makes it more widely applicable to a larger range of models than is usually considered in the literature. We considered particular models in which the mixing naturally arises (at least in part) from the charged lepton or right-handed neutrino sectors, and showed that these models share the same $R$ matrix as SD, and are hence in the same invariant class, i.e. they are just SD in disguise. We also discussed the application of our results to flavour-dependent leptogenesis where we show that the case of a real $R$ matrix is (approximately) realized in SD. Finally the connection of SD to the R-matrix makes it easier to identify in phenomenological studies.

In summary, the ISS approach amounts to the following proceedure. Starting from a particular see-saw model in a particular basis, one should determine the associated $R$ matrix, using either the standard approach involving the right-handed neutrino mass eigenvalues as in Eq[17, or using the $S$ matrix short-cut in Eq 20, useful when righthanded neutrino mass eigenvalues are not required. Having determined the invariant class $\mathcal{C}(R)$ to which it belongs, the particular model should properly be regarded as one member of an infinite number of other models related by basis transformations, and it can then easily be seen if any particular model is already present in the literature in a different guise. This also allows any given model to make contact with general phenomenological analyses based on the $R$ matrix. Although the ISS approach has been applied here to SD models, more generally it should prove to be a valuable model building tool in classifying and studying the myriad see-saw models that have been proposed in the literature.

\section{Acknowledgements}

I would like to thank Stefan Antusch, Michal Malinsky, Graham Ross and Ivo Varzielias for helpful discussions, and the CERN Theory Division for its hospitality and a Scientific Associateship. The author acknowledges support from the EU network MRTN 2004- 
503369.

\section{Appendix}

\section{A Two right-handed neutrino limit of sequential dom- inance}

In this Appendix we discuss the two right-handed neutrino limit of sequential dominance for the other cases not included in section 3.5.

The type of dominance in Eq[72] in the two right-handed neutrino limit corresponds to:

$$
\left(\begin{array}{lll}
\underline{v_{A}} & \underline{v_{B}} & \underline{0}
\end{array}\right)=\left(\begin{array}{lll}
\underline{0} & \underline{m}_{2}^{1 / 2} & \underline{m}_{3}^{1 / 2}
\end{array}\right) R_{A B 0}^{T}
$$

where

$$
R_{A B 0}^{T}=\left(R_{3}^{T}\right)_{s_{3}=1} R_{2}^{T}\left(R_{1}^{T}\right)_{s_{1}=-1}=\left(\begin{array}{ccc}
0 & 0 & -1 \\
c_{2} & s_{2} & 0 \\
s_{2} & -c_{2} & 0
\end{array}\right)
$$

where now $s_{3}=1, c_{3}=0, s_{1}=-1, c_{1}=0$ with $s_{2}, c_{2} \neq 0$. This results in:

$$
\begin{aligned}
& \underline{v_{A}}=c_{2} \underline{m}_{2}^{1 / 2}+s_{2} \underline{m}_{3}^{1 / 2} \\
& \underline{v_{B}}=s_{2} \underline{m}_{2}^{1 / 2}-c_{2} \underline{m}_{3}^{1 / 2}
\end{aligned}
$$

similar to Eq43 in the two family model, except that here the vectors have three components. SD here corresponds to $s_{2} \approx 1, c_{2} \approx 0$.

The type of dominance in Eq[73] in the two right-handed neutrino limit corresponds to:

$$
\left(\begin{array}{lll}
\underline{v_{A}} & \underline{0} & \underline{v_{B}}
\end{array}\right)=\left(\begin{array}{lll}
\underline{0} & \underline{m}_{2}^{1 / 2} & \underline{m}_{3}^{1 / 2}
\end{array}\right) R_{A 0 B}^{T}
$$

where

$$
R_{A 0 B}^{T}=\left(R_{3}^{T}\right)_{s_{3}=1} R_{2}^{T}\left(R_{1}^{T}\right)_{s_{1}=0}=\left(\begin{array}{ccc}
0 & -1 & 0 \\
c_{2} & 0 & -s_{2} \\
s_{2} & 0 & c_{2}
\end{array}\right)
$$

where now $s_{1}=0, c_{1}=1, s_{3}=1, c_{3}=0$ with $s_{2}, c_{2} \neq 0$. This results in:

$$
\begin{aligned}
& \underline{v_{A}}=c_{2} \underline{m}_{2}^{1 / 2}+s_{2} \underline{m}_{3}^{1 / 2} \\
& \underline{v_{B}}=-s_{2} \underline{m}_{2}^{1 / 2}+c_{2} \underline{m}_{3}^{1 / 2}
\end{aligned}
$$


similar to Eq43 in the two family model, except that here the vectors have three components. SD here corresponds to $s_{2} \approx 1, c_{2} \approx 0$.

The type of dominance in Eq[74 in the two right-handed neutrino limit corresponds to:

$$
\left(\begin{array}{lll}
\underline{v_{B}} & \underline{v_{A}} & \underline{0}
\end{array}\right)=\left(\begin{array}{lll}
\underline{0} & \underline{m}_{2}^{1 / 2} & \underline{m}_{3}^{1 / 2}
\end{array}\right) R_{B A 0}^{T}
$$

where

$$
R_{B A 0}^{T}=\left(R_{3}^{T}\right)_{s_{3}=1} R_{2}^{T}\left(R_{1}^{T}\right)_{s_{1}=1}=\left(\begin{array}{ccc}
0 & 0 & 1 \\
c_{2} & -s_{2} & 0 \\
s_{2} & c_{2} & 0
\end{array}\right)
$$

where now $s_{1,3}=1, c_{1,3}=0$ with $s_{2}, c_{2} \neq 0$. This results in:

$$
\begin{aligned}
& \underline{v_{B}}=c_{2} \underline{m}_{2}^{1 / 2}+s_{2} \underline{m}_{3}^{1 / 2} \\
& \underline{v_{A}}=-s_{2} \underline{m}_{2}^{1 / 2}+c_{2} \underline{m}_{3}^{1 / 2}
\end{aligned}
$$

similar to Eq43 in the two family model, except that here the vectors have three components. SD here corresponds to $s_{2} \approx 0, c_{2} \approx 1$.

The type of dominance in Eq[75] in the two right-handed neutrino limit corresponds to:

$$
\left(\begin{array}{lll}
\underline{v_{B}} & \underline{0} & \underline{v_{A}}
\end{array}\right)=\left(\begin{array}{lll}
\underline{0} & \underline{m}_{2}^{1 / 2} & \underline{m}_{3}^{1 / 2}
\end{array}\right) R_{B 0 A}^{T}
$$

where

$$
R_{B 0 A}^{T}=\left(R_{3}^{T}\right)_{s_{3}=1} R_{2}^{T}\left(R_{1}^{T}\right)_{s_{3}=0}=\left(\begin{array}{ccc}
0 & -1 & 0 \\
c_{2} & 0 & -s_{2} \\
s_{2} & 0 & c_{2}
\end{array}\right)
$$

where now $s_{3}=1, c_{3}=0, s_{1}=0, c_{1}=1$ with $s_{2}, c_{2} \neq 0$. This results in:

$$
\begin{aligned}
& \underline{v_{B}}=c_{2} \underline{m}_{2}^{1 / 2}+s_{2} \underline{m}_{3}^{1 / 2} \\
& \underline{v_{A}}=-s_{2} \underline{m}_{2}^{1 / 2}+c_{2} \underline{m}_{3}^{1 / 2}
\end{aligned}
$$

similar to Eq43 in the two family model, except that here the vectors have three components. SD here corresponds to $s_{2} \approx 0, c_{2} \approx 1$.

The type of dominance in Eq[76] in the two right-handed neutrino limit corresponds to:

$$
\left(\begin{array}{lll}
\underline{0} & \underline{v_{A}} & \underline{v_{B}}
\end{array}\right)=\left(\begin{array}{lll}
\underline{0} & \underline{m}_{2}^{1 / 2} & \underline{m}_{3}^{1 / 2}
\end{array}\right) R_{0 A B}^{T}
$$


where

$$
R_{0 A B}^{T}=\left(R_{3}^{T}\right)_{s_{3}=0}\left(R_{2}^{T}\right)_{s_{2}=0} R_{1}^{T}=\left(\begin{array}{ccc}
1 & 0 & 0 \\
0 & c_{1} & -s_{1} \\
0 & s_{1} & c_{1}
\end{array}\right)
$$

where now $s_{2,3}=0, c_{2,3}=1$ with $s_{1}, c_{1} \neq 0$. This results in:

$$
\begin{aligned}
& \underline{v_{A}}=c_{1} \underline{m}_{2}^{1 / 2}+s_{1} \underline{m}_{3}^{1 / 2} \\
& \underline{v_{B}}=-s_{1} \underline{m}_{2}^{1 / 2}+c_{1} \underline{m}_{3}^{1 / 2}
\end{aligned}
$$

similar to Eq43 in the two family model, except that here the vectors have three components. SD here corresponds to $s_{1} \approx 1, c_{1} \approx 0$.

\section{References}

[1] For a review see e.g. A. Strumia and F. Vissani, arXiv:hep-ph/0606054 R. N. Mohapatra et al., arXiv:hep-ph/0510213.

[2] For a recent review see e.g. J. W. F. Valle, arXiv:hep-ph/0608101.

[3] P. F. Harrison, D. H. Perkins and W. G. Scott, Phys. Lett. B 530 (2002) 167 arXiv:hep-ph/0202074; P. F. Harrison and W. G. Scott, Phys. Lett. B 535 (2002) 163 arXiv:hep-ph/0203209; P. F. Harrison and W. G. Scott, Phys. Lett. B 557 (2003) 76 arXiv:hep-ph/0302025]; an earlier related ansatz was proposed by: L. Wolfenstein, Phys. Rev. D 18 (1978) 958.

[4] For a review see e.g. S. F. King, Rept. Prog. Phys. 67 (2004) 107 arXiv:hep-ph/0310204; G. Altarelli and F. Feruglio, Springer Tracts Mod. Phys. 190 (2003) 169 arXiv:hep-ph/0206077; G. Altarelli, arXiv:hep-ph/0610164, R. N. Mohapatra and A. Y. Smirnov, arXiv:hep-ph/0603118.

[5] P. Minkowski, Phys. Lett. B 67 (1977) 421; M. Gell-Mann, P. Ramond and R. Slansky in Sanibel Talk, CALT-68-709, Feb 1979, and in Supergravity (North Holland, Amsterdam 1979); T. Yanagida in Proc. of the Workshop on Unified Theory and Baryon Number of the Universe, KEK, Japan, 1979; S.L.Glashow, Cargese Lectures (1979); R. N. Mohapatra and G. Senjanovic, Phys. Rev. Lett. 44 (1980) 912; J. Schechter and J. W. Valle, Phys. Rev. D 25 (1982) 774.

[6] C. Jarlskog, Phys. Rev. Lett. 55 (1985) 1039; C. Jarlskog, Z. Phys. C 29 (1985) 491; For a recent review see: C. Jarlskog, Phys. Scripta T127 (2006) 64 arXiv:hep-ph/0606050.

[7] J. A. Casas and A. Ibarra, Nucl. Phys. B 618 (2001) 171 arXiv:hep-ph/0103065. 
[8] S. F. King, Phys. Lett. B 439 (1998) 350 arXiv:hep-ph/9806440; S. F. King, Nucl. Phys. B 562 (1999) 57 arXiv:hep-ph/9904210; S. F. King, Nucl. Phys. B 576 (2000) 85 arXiv:hep-ph/9912492; S. F. King, JHEP 0209 (2002) 011 arXiv:hep-ph/0204360|; S. F. King, Phys. Rev. D 67 (2003) 113010 arXiv:hep-ph/0211228.

[9] A. Y. Smirnov, Phys. Rev. D 48 (1993) 3264 arXiv:hep-ph/9304205; G. Altarelli and F. Feruglio, JHEP 9811 (1998) 021 arXiv:hep-ph/9809596; G. Altarelli, F. Feruglio and I. Masina, Phys. Lett. B 472 (2000) 382 arXiv:hep-ph/9907532; I. Masina, Phys. Lett. B 633 (2006) 134 arXiv:hep-ph/0508031.

[10] S. F. King, JHEP 0508 (2005) 105 arXiv:hep-ph/0506297.

[11] S. F. King and G. G. Ross, Phys. Lett. B 520 (2001) 243 arXiv:hep-ph/0108112; S. F. King and G. G. Ross, Phys. Lett. B 574 (2003) 239 arXiv:hep-ph/0307190; G. Altarelli and F. Feruglio, arXiv:hep-ph/0504165; I. de Medeiros Varzielas, S. F. King and G. G. Ross, arXiv:hep-ph/0512313 S. F. King and M. Malinsky, arXiv:hep-ph/0608021; G. Altarelli, F. Feruglio and Y. Lin, arXiv:hep-ph/0610165.

[12] I. de Medeiros Varzielas and G. G. Ross, Nucl. Phys. B 733 (2006) 31 arXiv:hep-ph/0507176; I. de Medeiros Varzielas, S. F. King and G. G. Ross, arXiv:hep-ph/0607045.

[13] S. Antusch and S. F. King, Phys. Lett. B 631 (2005) 42 arXiv:hep-ph/0508044.

[14] S. Antusch and S. F. King, Phys. Lett. B 591 (2004) 104 arXiv:hep-ph/0403053; S. Antusch and S. F. King, Nucl. Phys. B 705 (2005) 239 arXiv:hep-ph/0402121; G. Altarelli, F. Feruglio and I. Masina, Nucl. Phys. B 689 (2004) 157 arXiv:hep-ph/0402155.

[15] S. Lavignac, I. Masina and C. A. Savoy, Nucl. Phys. B 633 (2002) 139 arXiv:hep-ph/0202086; I. Masina, arXiv:hep-ph/0210125 G. C. Branco, R. Gonzalez Felipe, F. R. Joaquim, I. Masina, M. N. Rebelo and C. A. Savoy, Phys. Rev. D 67 (2003) 073025 arXiv:hep-ph/0211001; S. Antusch, E. Arganda, M. J. Herrero and A. Teixeira, "Impact of theta(13) on lepton flavour violating processes within SUSY arXiv:hep-ph/0607263.

[16] A. Ibarra, JHEP 0601 (2006) 064 arXiv:hep-ph/0511136; A. Ibarra and G. G. Ross, Phys. Lett. B 591 (2004) 285 arXiv:hep-ph/0312138.

[17] S. Antusch, E. Arganda, M. J. Herrero and A. Teixeira, arXiv:hep-ph/0607263

[18] R. Barbieri, P. Creminelli, A. Strumia and N. Tetradis, Nucl. Phys. B 575 (2000) 61 arXiv:hep-ph/9911315; A. Abada, S. Davidson, F. X. Josse-Michaux, M. Losada 
and A. Riotto, JCAP 0604 (2006) 004 arXiv:hep-ph/0601083; E. Nardi, Y. Nir, E. Roulet and J. Racker, JHEP 0601, 164 (2006) arXiv:hep-ph/0601084.

[19] A. Abada, S. Davidson, A. Ibarra, F. X. Josse-Michaux, M. Losada and A. Riotto, arXiv:hep-ph/0605281, S. Pascoli, S. T. Petcov and A. Riotto, arXiv:hep-ph/0609125 G. C. Branco, R. G. Felipe and F. R. Joaquim, arXiv:hep-ph/0609297.

[20] S. Antusch, S. F. King and A. Riotto, arXiv:hep-ph/0609038. 